\title{
Statically Scoped Object Adaptation with Expanders
}

\author{
Alessandro Warth Milan Stanojević Todd Millstein \\ Computer Science Department \\ University of California, Los Angeles \\ \{awarth,milanst,todd $\} @$ cs.ucla.edu
}

\begin{abstract}
This paper introduces the expander, a new object-oriented (OO) programming language construct designed to support object adaptation. Expanders allow existing classes to be noninvasively updated with new methods, fields, and superinterfaces. Each client can customize its view of a class by explicitly importing any number of expanders. This view then applies to all instances of that class, including objects passed to the client from other components. A form of expander overriding allows expanders to interact naturally with $\mathrm{OO}$-style inheritance.

We describe the design, implementation, and evaluation of eJava, an extension to Java supporting expanders. We illustrate eJava's syntax and semantics through several examples. The statically scoped nature of expander usage allows for a modular static type system that prevents several important classes of errors. We describe this modular static type system informally, formalize eJava and its type system in an extension to Featherweight Java, and prove a type soundness theorem for the formalization. We also describe a modular compilation strategy for eJava, which we have implemented using the Polyglot extensible compiler framework. Finally, we illustrate the practical benefits of eJava by using this compiler in two experiments.
\end{abstract}

Categories and Subject Descriptors D.1.5 [Programming Techniques]: Object-oriented Programming; D.3.3 [Programming Languages]: Language Constructs and Features-Classes and objects

General Terms Design, Languages

Keywords expanders, object adaptation, modular typechecking

\section{Introduction}

Inheritance in object-oriented (OO) languages provides a form of extensibility for classes. A client of a class $C$ can use inheritance to easily create a customized version of $\mathrm{C}$ without requiring sourcecode access to or recompilation of $\mathrm{C}$. Further, the modular static type systems in mainstream languages like Java and C\# ensure that instances of this subclass can be safely used wherever a $C$ instance is expected.

However, several programming scenarios require forms of $o b$ ject adaptation, and today's OO languages do not easily support

Permission to make digital or hard copies of all or part of this work for personal or classroom use is granted without fee provided that copies are not made or distributed for profit or commercial advantage and that copies bear this notice and the full citation on the first page. To copy otherwise, to republish, to post on servers or to redistribute to lists, requires prior specific permission and/or a fee.

OOPSLA'06 October 22-26, 2006, Portland, Oregon, USA.

Copyright (C) 2006 ACM 1-59593-348-4/06/0010...\$5.00. this idiom. For example, consider a database of business objects, which a particular client wishes to display in an application-specific manner. The client likely needs to add new methods to these objects, and possibly to make these objects meet a client-specific interface. Inheritance would allow a new class to have these new behaviors but would do nothing to augment the existing objects. Design patterns like adapter and visitor [15] provide protocols that can be used to achieve the desired extensibility. However, these patterns are often tedious and error prone to implement, can require advance planning on the part of the original implementer of the class being extended, require invasive modifications whenever the adapted class hierarchy is augmented, and often rely on statically unsafe constructs like type casts.

In this paper, we introduce the expander, a new language construct that provides explicit support for object adaptation. An expander is a repository for adding new behaviors to existing classes and can include new methods, fields, and superinterfaces. Clients of an expander may make use of these additional behaviors on any object of the original class (or a subclass), including objects passed to the client from other components. Expanders also interact in a natural way with inheritance: expanders can be overridden for particular subclasses, and message sends dynamically dispatch to the best method for the given receiver, as with traditional message sends.

There is a large body of research into mechanisms for increasing the extensibility of classes. Expanders are distinguished by a novel combination of design properties that are targeted to support object adaptation:

In-place adaptation. An expander updates an existing class with new behaviors, rather than creating a new class that contains the additional behaviors. This design choice is critical for support of object adaptation. In particular, the new behaviors in an expander are available for use on instances of the original class, regardless of where or when those instances were created.

This design contrasts with the variety of proposals for flexible class extensibility, including mixins [5, 13], traits [28], proposals that include a form of dependently typed classes [10, 11, 21, 23], and parameterized module systems for OO languages $[12,20]$. These works have a very different goal from ours and are quite complementary. The customizations supported by these languages provide for fine-grained forms of code reuse across classes and class hierarchies but do nothing to adapt existing objects. Indeed, typically some advance planning, for example via the factory design pattern [15] or a form of module parameterization, is required to ensure that the full customization of a class can be produced before any instances are created. In contrast, expanders support object adaptation and allow multiple clients to view the same object in different ways, but they do not provide code reuse across classes and class hierarchies.

Statically scoped adaptation. Expanders target the problem of adapting existing classes to the needs of new clients, without affecting the behavior of existing clients. This focus led us to a 
design whereby each client explicitly imports the expanders that are needed in order to perform its task; all other expanders are out of scope and cannot affect the client's behavior. In addition to making each client's behavior easier to reason about, this static scoping of expanders naturally allows an object to be "expanded" in different ways by different clients in the same program without any conflict.

This design contrasts with the inter-type declarations in aspectoriented languages like AspectJ [19], which also allow existing classes to be augmented with new methods, fields, and superinterfaces. While aspects can be used for object adaptation, such adaptation affects all clients of a class implicitly, including existing ones. This power can be quite useful, but it also makes it difficult to understand how a given client will behave without global knowledge of all aspects in the program.

Modular type safety. The existence of multiple versions of a class has the potential to cause erroneous or undesirable behavior at run time, whereby a field or method of one version is expected but another is actually used. This problem is exacerbated in the presence of inheritance, since subclasses of the original class may add behaviors that clash with those added by an expander. Aside from violating programmer expectations, these kinds of problems also potentially compromise type safety, for example if a field of one type is expected but a field of a different type is actually used.

Because of the static scoping of expanders, it is possible to perform modular static checking to guarantee type safety in the presence of expanders. A class that passes these modular checks cannot suffer from the kinds of errors described above, regardless of what unknown classes and expanders are ultimately linked into the final application. To our knowledge, this guarantee of modular type safety is unique among languages that are expressive enough to support object adaptation, including the AspectJ language described above and the recent work on Classboxes [4, 3]. MultiJava $[7,6]$ provides modular typechecking for the addition of new methods to existing classes. However, MultiJava does not allow new fields or superinterfaces to be added to existing classes, so it is unable to express many object adaptation scenarios.

We have instantiated our notion of expanders in the language eJava, a backward-compatible extension to Java. We have designed a modular type system for eJava as well as a modular compilation strategy from eJava to Java, and we have implemented both in the Polyglot extensible compiler framework [22]. We have also formalized eJava as an extension of Featherweight Java (FJ) [18] that we call Featherweight eJava ( $\mathrm{FeJ})$, and we have proven its modular type system sound.

Finally, we have used our eJava implementation to gain experience with the language and gauge its practical utility. First, we used eJava to build a small application that employs Java's Swing library to display some existing business objects in an application-specific manner, and we compare the benefits and limitations against an implementation in pure Java. Second, we performed an exploratory study on the Eclipse integrated development environment [8]. We identify several Java-based extensibility idioms used in the implementation of Eclipse, and we explore the ways in which expanders can allow these idioms to be more naturally and reliably expressed.

The rest of the paper is structured as follows. Section 2 introduces expanders by a number of examples. Section 3 details eJava's method-lookup semantics and associated static typechecks. Section 4 describes the FeJ formalism, which provides a more precise description of the language and its modular type system. Section 5 describes the modular compilation strategy for eJava, as implemented in the eJava compiler. Section 6 discusses our two experiments with the language. Section 7 compares expanders with related work, and Section 8 concludes.

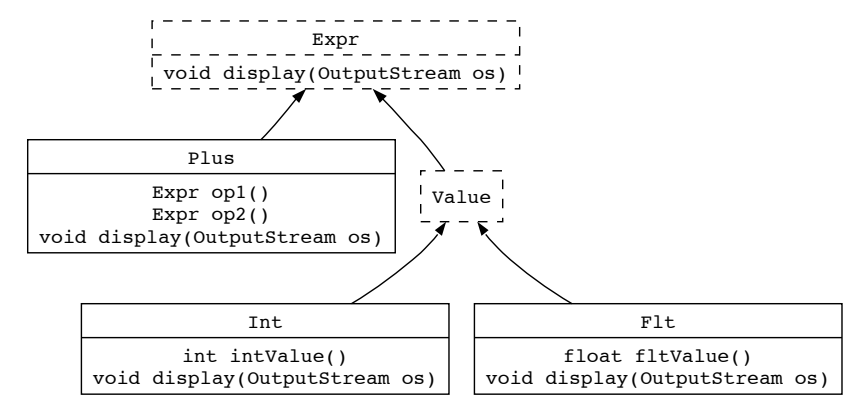

Figure 1. An expression hierarchy.

\section{Expanders}

This section informally describes the capabilities of expanders and their interaction with the other features of Java. As a running example, consider a Parser class that parses a simple language of expressions supporting integers, floating-point numbers, and addition. The Parser has a parse method that accepts a String and produces an abstract syntax tree (AST), which is represented using the Expr class hierarchy shown in Figure 1. We illustrate eJava's expressiveness and flexibility by considering a number of ways in which clients may wish to employ this parser as part of a larger application. To reuse the parser, clients must first customize it to their needs. eJava allows each client's customizations to be expressed without modifying or requiring access to the source code of the parser or the Expr hierarchy, without requiring any advance planning by the implementer of the parser, and without interfering with the customizations of other clients.

\subsection{Noninvasive Visitors with Expanders}

It is natural for clients to wish to augment the Expr hierarchy with new methods, in order to perform new passes over the AST. For example, suppose a client wishes to add an eval method that evaluates an expression and returns the resulting value. One approach would be to create a subclass EvalExpr of Expr that includes such a method, as well as new classes like EvalPlus and Evalint. However, the lack of multiple implementation inheritance in Java would require this approach to duplicate a significant amount of code. For example, EvalP lus must inherit from EvalExpr in order to override its eval method, but then it cannot also inherit from Plus, forcing the client to duplicate all of the existing capabilities of addition expressions. Further, this approach would not allow the existing Parser class to be reused, since it creates instances of the original expression classes rather than the new ones.

The standard approach to this problem is the visitor design pattern [15], which allows clients to easily add new functionality to existing classes externally. However, this approach requires the original implementer of the expression hierarchy to have anticipated the need for visitors, by providing a Visitor class and the appropriate accept methods for each kind of expression. The visitor pattern also has other, more minor, problems, for example the fact that every external operation must have the same argument and result types.

Therefore, clients are often forced to add new functionality using utility classes such as Evaluator, shown in Figure 2. While this approach allows the new "method" to be added without modifying existing code, it has several drawbacks. Since the method is placed in the Evaluator class, dynamic dispatch on the Expr instance must be performed manually via runtime type tests and type casts. These type tests must be executed in the correct order (from mostto least-specific), which is easy to get wrong for large and complex type hierarchies. Further, using utility classes like Evaluator 


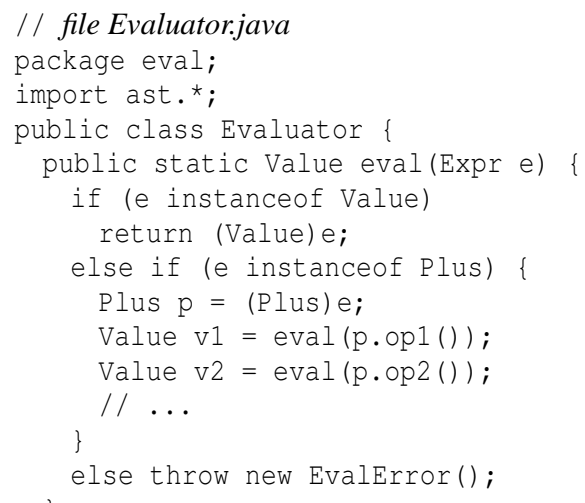

Figure 2. An external evaluator for Exprs in Java.

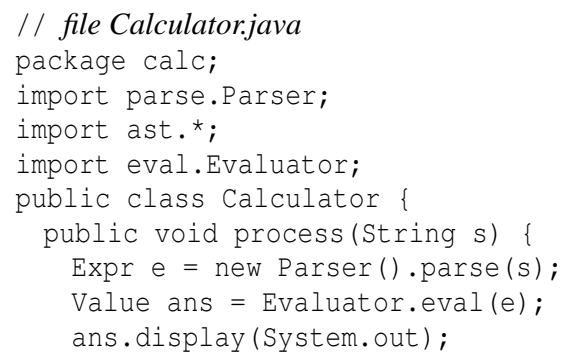

Figure 3. A client of the evaluator in Figure 2.

forces clients to distinguish syntactically between calls to external methods of Expr like eval and calls to ordinary methods of the hierarchy like display. This difference is illustrated by the sample client in Figure 3, which implements a calculator application using Parser and Evaluator.

Expanders in eJava provide a natural solution to these problems. An expander is a repository for augmenting an existing class in several different ways, including the introduction of new methods, without requiring source-code access to the original class. For example, an expander that adds an eval method to Expr would look as follows:

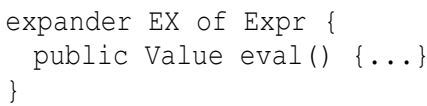

This expander is given the name EX and is declared to expand the Expr class. The body of the expander then provides the new eval method, just as it would be declared in the original class. For example, the method has an implicit receiver argument of type Expr which can be referenced as this in the method's body. An expander can declare any number of new methods.

We could choose to implement the semantics of expression evaluation entirely within the eval method of the above expander, analogous to the code in Figure 2. However, expanders support a better solution through expander overriding, as shown in Figure 4. In addition to the expander for Expr, the compilation unit (i.e., file) shown includes two expanders that provide overriding implementations of eval for particular subclasses of Expr. We say that the three expanders are in the same expander family, which is a collec-

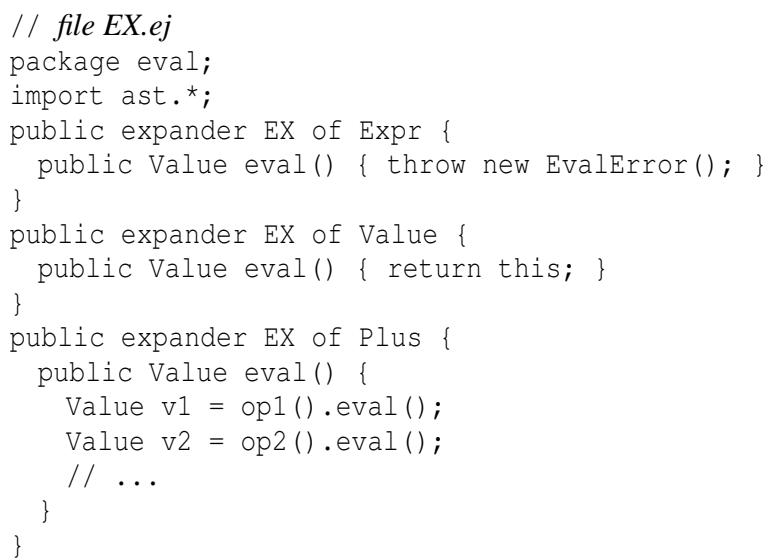

Figure 4. An external evaluator for Exprs in eJava.

tion of expanders of the same name. Each expander family has a unique top expander: all other expanders in the family augment a class that is a subclass of the top expander's class. Therefore, the first expander in the figure is the top expander for EX.

The code in Figure 4 has the same behavior as the eval method in Figure 2. However, the use of expander overriding obviates the need for instanceof tests and type casts. Instead, the method implementations make use of Java's ordinary dynamic dispatch on the receiver argument, just as if they were declared in the original expression classes.

We allow methods in expanders to override methods in other expanders, as the example shows. However, a method in an expander cannot override an ordinary method of the associated class. This restriction is the result of a tradeoff between modular compilation and external method overriding that has been previously recognized [6]. ${ }^{1}$ Further, we disallow overriding expanders from declaring members that are not declared in the top expander. This simplification ensures that an expander family presents a unique "interface" to clients, and it results in no loss of expressiveness since a separate expander family can be created to contain any new members desired for a particular class and its subclasses.

An expander does not have privileged access to its associated receiver class, instead obeying the same visibility rules as other clients of the class. This restriction ensures that an expander does not break any internal invariants of existing classes. An expander as well as its members can have the usual Java privacy modifiers. These modifiers are interpreted relative to the expander's location, rather than that of the original class. For example, a method with package visibility in an expander is only accessible within the package in which that expander (not the class it expands) was declared.

Figure 5 shows the new version of our calculator application that uses EX (instead of Evaluator). In order to make use of the "expanded" view of the expression hierarchy, the expander family EX must be explicitly used, with a syntax analogous to Java's import statement. The methods declared for the EX expander family are thereby made available for use, via the same syntax as if they were declared in the original classes. This is illustrated by the invocation e.eval () in the figure. Without the use statement, that invocation would fail to typecheck, since Expr does not originally support an eval method.

\footnotetext{
${ }^{1}$ An expander is still allowed to statically overload methods of the associated class.
} 


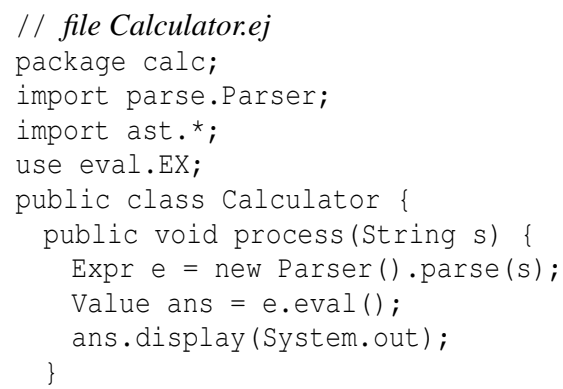

Figure 5. A client of the evaluator in Figure 4.

The explicit import of expanders via use enables modular reasoning about clients. For example, any client of the expression hierarchy that does not use EX can be safely typechecked without knowledge of the EX expander family. Further, there can exist multiple expander families in a program that augment the expression hierarchy with an eval method. Each client in the program can use the version of expression evaluation that suits its needs, without being affected by the other versions. The rules for method lookup, as well as our static typechecks to ensure that method lookup will always succeed at run time, are described in Section 3.

An expander can be used to add functionality to any class, including final classes. For example, one could implement an expander of Java's String class that adds a helper method like removeWhitespace(). Clients can then use this expander and invoke removeWhitespace on any value of type String, including string literals. Because an expander does not have privileged access to the class being expanded, this expander cannot violate any of the internal invariants of Java's String class.

\subsection{Adapting with Expanders}

While the ability to add methods to existing classes from the outside is very useful, often it is not enough on its own. For example, suppose that our Calculator class is to be used within an application in which all console output must be enqueued in a printing thread via the enqueue () method of the Printer class:

public static void enqueue (Printable p) $\{\ldots\}$

This method takes an instance of the Printable interface, which is declared as follows:

public interface Printable \{ void print(); \}

In Java, a programmer could use the adapter design pattern [15] to adapt existing instances of Expr and subclasses to support the Printable interface. To do so, the programmer would create a wrapper class PrintableExpr for Expr that meets the Printable interface and implements the associated methods by appropriately forwarding to the methods of the underlying expression. Whenever an instance of Expr or a subclass needs to be treated as Printable, it would first be adapted by explicitly constructing an instance of PrintableExpr, passing the adaptee object as a parameter.

Expanders provide a simpler and more natural solution to this problem, as shown in Figure 6. An expander can use the implements clause to declare that the class being expanded (and its subclasses) implements any number of new interfaces. An interface can be implemented by a combination of methods already declared or inherited in the original class and methods declared in the expander. Overriding expanders can also be used in conjunction with the ability to meet new interfaces. For example, we could

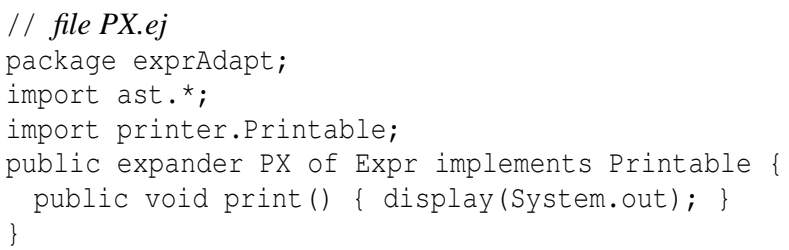

Figure 6. Adapting Exprs to Printables with expanders.

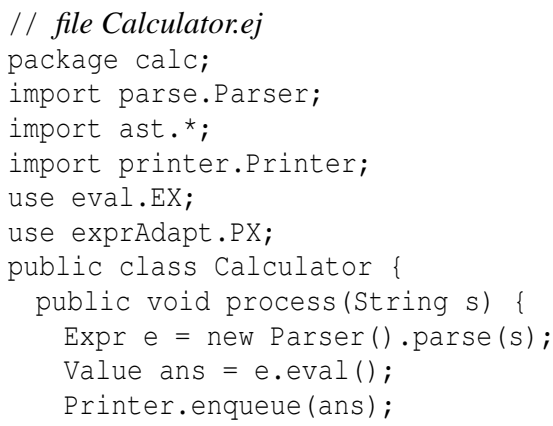

Figure 7. A fancier calculator, which uses both evaluation and printing functionality.

provide an overriding expander to define print specially for Plus expressions.

Figure 7 shows an upgraded version of our Calculator class, which uses both evaluation and printing functionality. The compilation unit now uses both EX and PX, allowing Expr instances to be viewed as containing both eval and print methods, as well as meeting the Printable interface. Since Value is a subclass of Expr, the ans instance of Value is a typesafe argument to enqueue, without requiring any explicit adaptation as would be necessary with the adapter design pattern. It is important to note that the implementer of the Printer class need not know about the existence of either the Expr hierarchy or its PX expander. All that the enqueue method of Printer requires is that the given argument meets the Printable interface; our modular compilation strategy described in Section 5 ensures that this requirement is satisfied.

\subsection{Adding State with Expanders}

Expanders can also be used to add state to existing classes. An example is shown in Figure 8, which augments the expression hierarchy with type information. A type field is added to Expr (and subclasses) to store an expression's type, along with a getter method and a method to compute the type.

\subsection{Expanding Interfaces}

Expanders can also be used to add functionality to interfaces. For example, Figure 9 uses an expander to add a printAll method to instances of Java's Iterable interface, which prints all elements of the iterable collection. The expander makes use of the iterator method defined for Iterable.

An expander for an interface is not tied to a particular class hierarchy. Instead, it can be used to expand any class that implements the specified interface. This interface acts as the abstract "requirements" of that expander, so the expander can be considered to be parameterized by the classes that it expands. 


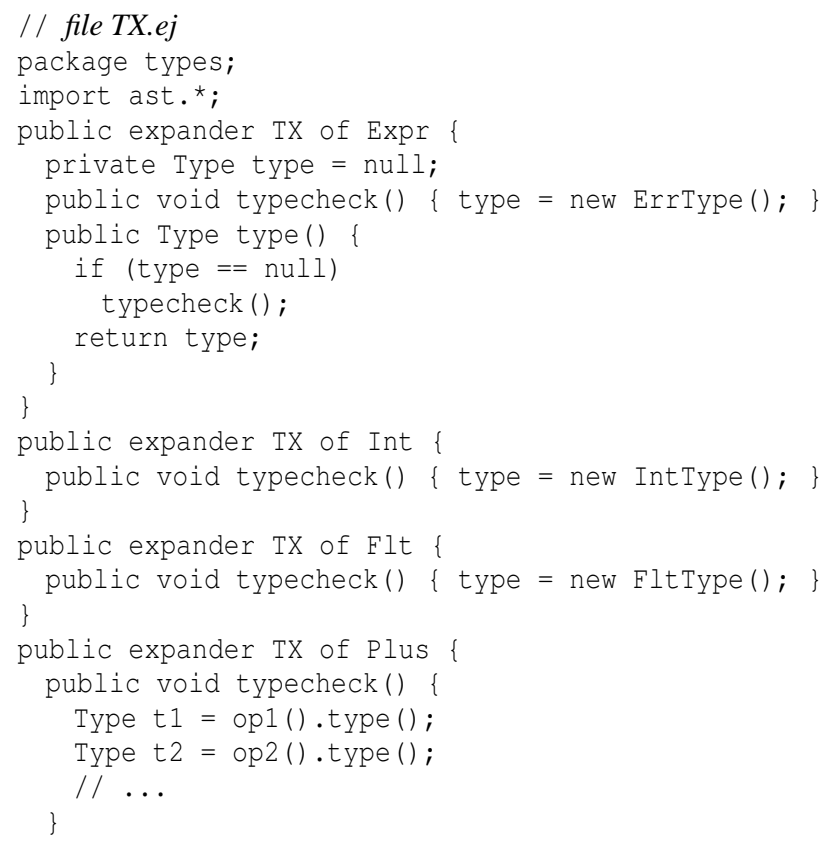

Figure 8. Adding state in an expander.

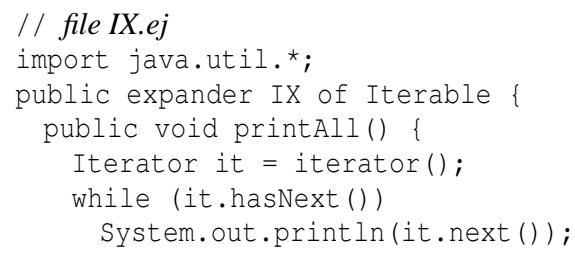

Figure 9. An expander for an interface.

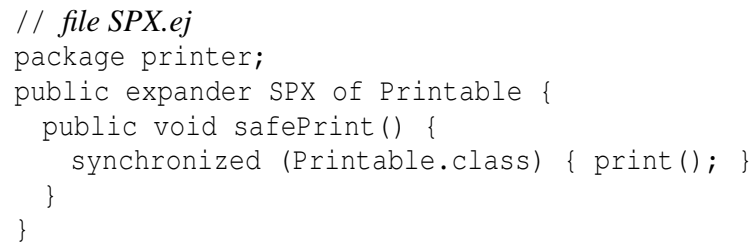

Figure 10. SPX, the safe printing expander.

Further, other expanders can be used to increase the applicability of an interface-based expander. As shown earlier, an expander can adapt an existing class to support a new interface. This capability can therefore be used to allow an existing class to meet the requirements of an interface-based expander.

As an example, Figure 10 shows SPX, the safe printing expander, which augments instances of the Printable interface with "safe-printing" functionality (the safePrint method uses the Printable class as a lock to ensure that no two Printable objects are ever printed to the console at the same time). Although Expr does not originally implement Printable, its instances can be aug-

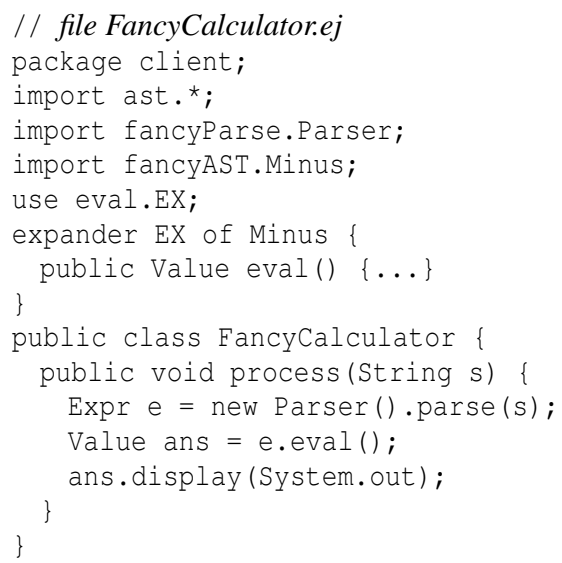

Figure 11. Adding a new expander to an existing expander family.

mented by SPX provided we first adapt them to the Printable interface by using PX, the Printable expander from Figure 6.

\subsection{Locally Augmenting Expander Families}

In the examples so far, all expanders in a given family were declared in the same compilation unit. However, it is possible for an expander family's associated class hierarchy to be augmented by clients with new subclasses. When this happens, it is often desirable to augment the expander family with overriding expanders to provide special-purpose behavior for these classes. To this end, eJava allows a client of an expander family to locally augment that family with overriding expanders for new classes. The additional expanders are hygienic: they only affect the expander family's behavior within the current compilation unit.

For example, suppose a client of the original expression hierarchy adds a subclass Minus of Expr and creates a new parser to handle subtraction. Figure 11 shows how a client of the EX expander can update it to handle Minus appropriately. The call to eval in FancyCalculator will dynamically dispatch to the new eval implementation whenever the receiver is an instance of Minus.

\section{Method Lookup and Modular Typechecking}

eJava's rules for method lookup are a natural generalization of those of Java. At compile time, each message send expression in a program is determined to refer to a unique method family, or else a static error is signaled. At run time, the most-specific method for the given receiver argument in the statically determined method family is selected and invoked. We discuss each of these phases in turn, along with the corresponding static checks to ensure that method lookup always succeeds at run time. The section ends by illustrating a type hole that can arise in other languages for object adaptation, which our semantics for method lookup safely avoids.

\subsection{Static Method Family Selection}

Each method in eJava, and in Java, can be thought of as belonging to a unique method family. The method family of a method $m_{1}$ is determined as follows. If $m_{1}$ overrides a method $m_{2}$, then $m_{1}$ is in the same method family as $m_{2}$. Otherwise, $m_{1}$ belongs to a different method family. The Java typechecker statically ensures that there is a single best method family for each message send expression in a program, as discussed in the Java Language Specification [16], $\S 15.12$. In the presence of static overloading, a method family is essentially defined by the method's name, number of arguments, and static argument types. An error is signaled if either there are no 
applicable method families for a given message send or there are multiple applicable families but no most-specific one.

In eJava, we first use Java's rules to find a most-specific method family for a message send expression, completely ignoring expanders. If a unique such method family exists, we are done. If there are multiple such families but no most-specific one, then an ambiguity error occurs, as in Java. However, if there are no applicable method families, we then search for a unique applicable method family in the expanders that are used by the current compilation unit, signaling a compile-time error otherwise. As in Java, the method family associated with a method in an expander is defined by the method's name, number of arguments, and static argument types, and additionally by the name of the enclosing expander. The rules for statically determining to which field declaration a field access refers are analogous.

To illustrate these rules, consider statically determining the method family for the invocation e.eval () in Figure 5:

- Given the definitions of the Expr class (Figure 1) and the EX expander family (Figure 4), the invocation is statically determined to invoke the method family consisting of the three eval methods in the EX expanders.

- If the use EX; statement were omitted from Figure 5, the eJava typechecker would signal a static error, since there is no applicable method family for the invocation.

- If the original Expr class contained a (possibly abstract) zeroargument eval method, then that method's associated family would be selected, even in the presence of the use EX; statement.

- Suppose there existed another expander EX2 that also defines a zero-argument eval method for Expr. If Figure 5 were augmented to include the statement use EX2; the eJava typechecker would signal a static error, since the eval method families in EX and EX2 are ambiguous. However, the error is only signaled because of the call to eval. If that call were removed, EX and EX2 could both be used without problems, allowing other members of these expanders to be accessed.

Finally, we also provide a mechanism for the programmer to explicitly specify the intended method family, which is useful for handling ambiguous situations. If the receiver in a method invocation has the form expr with X, where expr is an eJava expression and $X$ is an expander name, then only the expander $X$ is searched for an appropriate method family; neither the original class of the receiver nor any other expanders are considered. For example, in the scenario described in the last bullet above, the programmer can cause static method-family selection to succeed by explicitly declaring which expander is intended, e.g., (e with EX2) .eval (). The with expression can also be used to multiply expand an expression. For example, the expander SPX can be used to adapt an expression e of type Expr via the syntax (e with PX) with SPX, thereby allowing the safePrint method to be invoked.

\subsection{Dynamic Method Selection}

Run-time method lookup in eJava is defined as in Java: the dynamic class of the receiver argument is used to find the most-specific applicable method from the statically determined method family, and that method is then invoked. For example, if the run-time class of $e$ in Figure 5 is Plus, then the first and third eval methods in Figure 4 are applicable, and the latter is the most-specific applicable method. Any local overriding expanders are taken into account when a client invokes a method family in an expander, allowing the new methods to be dispatched to appropriately.

As part of modular static typechecking of each class, Java ensures that each method family is exhaustive and unambiguous, thereby guaranteeing that run-time method lookup on that family will always succeed. For example, Java checks that a concrete class C declares or inherits a concrete method for every method family declared in a superinterface or superclass. This check ensures exhaustiveness: instances of $C$ and its subclasses are guaranteed to have at least one applicable method in each inherited method family. Java lacks multiple inheritance of classes, so there is no possibility of run-time ambiguities.

eJava includes additional modular static requirements on expanders to ensure that run-time method lookup always succeeds. First, an expander cannot contain an abstract method, even if the class being expanded is abstract. This requirement ensures exhaustiveness in the presence of any unknown concrete subclasses of that class, which must always be assumed to exist given only a modular view of the program. Second, an overriding expander can only expand a class, not an interface. This requirement prevents ambiguities that are not modularly detectable, which can occur if an unknown class implements multiple interfaces. Both requirements are analogous to requirements on MultiJava's open classes [7], which also allow methods to be defined external to their classes.

\subsection{Preventing Accidental Method Overriding}

Our method lookup rules rely on the way in which a program's methods are partitioned into method families. A key property of eJava is the ability to modularly determine the method family to which a method belongs. A method's associated method family is defined to be the same as that of any method that it overrides, and the methods that a particular method overrides can be determined based on information that is available in the static scope where the method is declared. This property of eJava is shared by Java, but it is not shared by other languages that support forms of object adaptation, including AspectJ [19] and Classboxes [4, 3].

To illustrate this distinction, consider again the Expr class hierarchy (Figure 1) and the EX expander family (Figure 4). As mentioned earlier, if another expander EX2 for Expr also defines an eval method, this method (and any eval methods in overriding expanders for EX2) is considered to belong to a different method family from the eval methods for EX. If these methods were all considered part of the same method family, there could be run-time ambiguities that are not modularly detectable, for example because there are two eval methods defined for the class Expr.

Worse, there may be no ambiguity for a given receiver argument to eval, but clients will simply get unexpected behavior at run time. For example, suppose EX2 defines an eval method for some subclass SpecialPlus of Plus. If the EX and EX2 eval methods were all considered part of the same method family, a client that uses EX and invokes eval on an instance of Specialplus would unexpectedly execute the method from EX2. Aside from potentially having the wrong behavior, this situation is not modularly typesafe, since for example the eval methods in EX2 could well have a different return type from those in EX. Since neither EX nor EX2 is aware of the other modularly, this clash eludes modular typechecking.

Analogous conflicts can occur between a class and an expander, instead of between two expanders. For example, consider a client of a class $C$ that creates an expander containing a print method and uses this method to display an array of Cs in an applicationspecific way. By our semantics, this print method is considered to be in its own method family. Therefore, even if the array contains some instances of $D$, a subclass of $C$ that happens to provide its own print method, the client will behave as expected, using only the expander's implementation of print. If the two print methods were considered to be part of the same method family, then D's print method would be invoked and our application would behave incorrectly, since D's print method likely does not conform to the client's application-specific display format. 


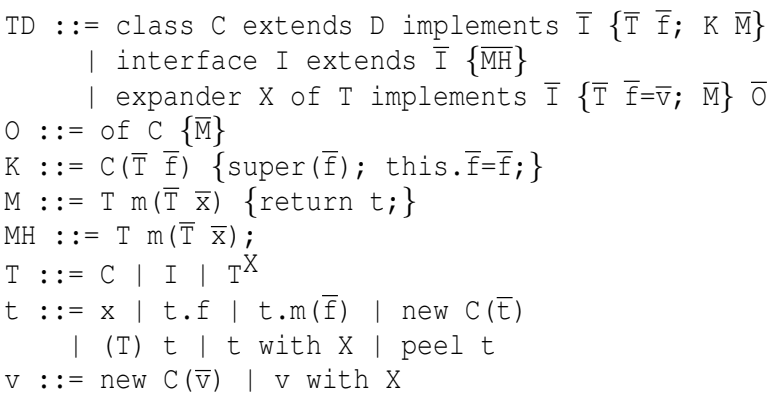

Figure 12. The syntax of Featherweight eJava.

To our knowledge, eJava is the only language for object adaptation that modularly ensures the absence of these accidental overriding errors, and hence modularly ensures type safety. Two aspects in AspectJ that define the same method for a given class can easily cause such conflicts, and similarly for two classboxes that refine a given class in the same way. These errors can only be detected with global knowledge of all aspects or classboxes in the program that can affect a given class. For example, in the Classbox/J implementation, clashes involving incompatible return types are only detected by the regular Java typechecker, which runs after the Classbox/J compiler weaves all refinements in the program for a given class into that class's declaration. Accidental overriding that does not violate type safety, such as the print example above, is silently allowed by Classbox/J, causing potentially unintended behavior at run time. The next section formalizes our modular type system for eJava and proves its soundness.

\section{Featherweight eJava}

This section describes Featherweight eJava (FeJ), an extension of Featherweight Java (FJ) [18] that formalizes our notion of expanders and its associated modular type system. Aside from making the eJava language semantics precise, FeJ also allows us to prove a type soundness theorem, which validates the sufficiency of our modular type system for ruling out run-time type errors, including problems with accidental overriding. The full details of FeJ and its type soundness proof are available in our companion technical report [33]

\subsection{Syntax}

The syntax of FeJ is shown in Figure 12. We use notational conventions and sanity conditions analogous to those of FJ. For example, the syntax $\bar{D}$ denotes a sequence of zero or more elements of the domain D. Also, an FeJ program consists of a type table TT, which maps class, interface, and expander names to their associated declarations, and an expression. We comment on other conventions as necessary throughout this section.

FeJ augments FJ with interfaces and expanders. For simplicity, the expander declaration both provides the top expander and all overriding expanders (via the $\overline{0}$ portion of the declaration); local overriding expanders are not modeled. The eJava language largely infers where "expansion" must occur as part of static method family selection, as described in the previous section. FeJ programs are explicit about expander usage: there are no use declarations, an object is expanded via the with expression, and an expanded object is "unexpanded" via the peel expression. Similarly, we include an explicit type $\mathrm{T}^{\mathrm{X}}$ for objects of type $\mathrm{T}$ that are expanded by expander $\mathrm{x}$.

$$
\begin{aligned}
& \mathrm{T}<: \mathrm{T} \\
& \frac{\mathrm{S}<: \mathrm{T} \quad \mathrm{T}<: \mathrm{U}}{\mathrm{S}<: \mathrm{U}} \\
& \frac{T T(\mathrm{C})=\text { class } \mathrm{C} \text { extends } \mathrm{D} \text { implements } \overline{\mathrm{I}}\{\ldots\}}{\mathrm{C}<: \mathrm{D}} \\
& \frac{T T(\mathrm{C})=\text { class } \mathrm{C} \text { extends } \mathrm{D} \text { implements } \overline{\mathrm{I}}\{\ldots\}}{\mathrm{C}<\mathrm{I}_{i}} \\
& \frac{T T(\mathrm{I})=\text { interface } \mathrm{I} \text { extends } \overline{\mathrm{J}}\{\ldots\}}{\mathrm{I}<: \mathrm{J}_{i}} \\
& \frac{T T(\mathrm{X})=\text { expander } \mathrm{X} \text { of } \mathrm{T} \text { implements } \overline{\mathrm{I}}\{\ldots\} \overline{\mathrm{O}}}{\mathrm{T}^{\mathrm{X}}<: \mathrm{I}_{i}} \\
& \frac{\mathrm{S}<: \mathrm{T}}{\mathrm{S}^{\mathrm{X}}<: \mathrm{T}^{\mathrm{X}}}
\end{aligned}
$$

Figure 13. Subtyping in FeJ.

\subsection{Subtyping}

FeJ's subtyping judgment formalizes the relationship between expanded and unexpanded objects; it is shown in Figure 13. The first three rules are the adaptation of FJ's subtyping rules to our context. The following two rules handle subtyping in the presence of Javastyle interfaces appropriately. The next rule is the essence of object adaptation: an expanded object can be typed with any interface that is implemented by the associated expander, allowing the expanded object to be passed wherever values meeting that interface are expected. The final rule extends this ability for object adaptation to instances of any subtype of the type being expanded in an expander declaration.

A notable absence from the subtyping relation is the axiom $\mathrm{T}^{\mathrm{X}}<$ : T. Omitting this rule forces an expanded object to be unexpanded via peel before it is passed where a value of the original type is expected. It also ensures that methods in an expander will not be treated as overriding methods in the unexpanded type. Both of these behaviors mirror the semantics and implementation strategy of the eJava language. Despite the absence of this subtyping relationship, values of type $\mathrm{T}^{\mathrm{X}}$ may still access methods and fields of the original type $\mathrm{T}$, as we show below. In this way, for example, an expanded type may "inherit" methods from the original type in order to meet a new interface.

\subsection{Dynamic Semantics}

Figure 14 provides the small-step operational semantics of FeJ and makes use of the helper rules in Figure 15. We only present the rules that relate to expanders; the rest of $\mathrm{FeJ}$ is identical to FJ. The first two rules define field lookup for expanded objects. If the expander defines the field being accessed, then its associated value is returned. Otherwise, field lookup proceeds in the unexpanded object.

The rules for method lookup on expanded objects are described next, and they depend on the mbody helper function, which finds the best implementation of the method $m$ for class $C$ in expander 


$$
\begin{aligned}
& \frac{\operatorname{fields}(\mathrm{X})=\overline{\mathrm{T}} \overline{\mathrm{f}}=\overline{\mathrm{v}}}{\left(\mathrm{v} \text { with X) } \mathrm{f}_{i} \longrightarrow \mathrm{v}_{i}\right.} \\
& \frac{\operatorname{fields}(\mathrm{X})=\overline{\mathrm{T}} \overline{\mathrm{g}}=\overline{\mathrm{v}} \quad \mathrm{f} \notin \overline{\mathrm{g}}}{(\mathrm{v} \text { with X) } \mathrm{f} \longrightarrow \mathrm{v} \cdot \mathrm{f}} \\
& \frac{\mathrm{v}=\text { new } \mathrm{C}(\overline{\mathrm{v}}) \quad \operatorname{mbody}(\mathrm{m}, \mathrm{X}, \mathrm{c})=\left(\overline{\mathrm{x}}, \mathrm{t}_{0}\right)}{(\mathrm{v} \text { with } \mathrm{X}) \cdot \mathrm{m}(\overline{\mathrm{u}}) \longrightarrow[\overline{\mathrm{x}} \mapsto \overline{\mathrm{u}}, \text { this } \mapsto \mathrm{v} \text { with } \mathrm{x}] \mathrm{t}_{0}} \\
& \frac{\mathrm{v}=\mathrm{v}^{\prime} \text { with } \mathrm{X}^{\prime} \quad \operatorname{mbody}(\mathrm{m}, \mathrm{x}, \text { object })=\left(\overline{\mathrm{x}}, \mathrm{t}_{0}\right)}{(\mathrm{v} \text { with } \mathrm{X}) \cdot \mathrm{m}(\overline{\mathrm{u}}) \longrightarrow[\overline{\mathrm{x}} \mapsto \overline{\mathrm{u}}, \text { this } \mapsto \mathrm{v} \text { with } \mathrm{x}] \mathrm{t}_{0}} \\
& T T(\mathrm{X})=\text { expander } \mathrm{X} \text { of } \mathrm{T} \text { implement } \mathrm{S} \quad\{\overline{\mathrm{T}} \overline{\mathrm{f}}=\overline{\mathrm{V}} ; \overline{\mathrm{M}}\} \overline{\mathrm{O}} \\
& m \text { is not defined in } \bar{M} \\
& (\mathrm{v} \text { with X) } \cdot \mathrm{m}(\overline{\mathrm{u}}) \longrightarrow \mathrm{v} \cdot \mathrm{m}(\overline{\mathrm{u}}) \\
& \text { peel (v with } \mathrm{X}) \longrightarrow \mathrm{v} \\
& \frac{t_{0} \longrightarrow t_{0}^{\prime}}{t_{0} \text { with } x \longrightarrow t_{0}^{\prime} \text { with } x} \\
& \frac{t_{0} \longrightarrow t_{0}^{\prime}}{\text { peel } t_{0} \longrightarrow \text { peel } t_{0}^{\prime}}
\end{aligned}
$$

Figure 14. FeJ evaluation rules.

$$
\begin{aligned}
& \operatorname{fields}(\mathrm{X})=\overline{\mathrm{T}} \overline{\mathrm{f}}=\overline{\mathrm{V}} \\
& T T(\mathrm{X})=\text { expander } \mathrm{X} \text { of } \mathrm{T} \text { implements } \overline{\mathrm{I}}\{\overline{\mathrm{T}} \overline{\mathrm{f}}=\overline{\mathrm{V}} ; \overline{\mathrm{M}}\} \overline{\mathrm{O}} \\
& \text { fields }(\mathrm{X})=\overline{\mathrm{T}} \overline{\mathrm{f}}=\overline{\mathrm{V}} \\
& \operatorname{mbody}(\mathrm{m}, \mathrm{X}, \mathrm{C})=(\overline{\mathrm{x}}, \mathrm{t}) \\
& T T(\mathrm{X})=\text { expander } \mathrm{X} \text { of } \mathrm{T} \text { implements } \overline{\mathrm{I}}\{\overline{\mathrm{T}} \overline{\mathrm{f}}=\overline{\mathrm{V}} ; \overline{\mathrm{M}}\} \overline{\mathrm{O}} \\
& \text { of } \left.\mathrm{C}\left\{\overline{\mathrm{M}^{\prime}}\right\} \in \overline{\mathrm{O}} \quad \mathrm{U} \mathrm{m}(\overline{\mathrm{U}} \overline{\mathrm{x}}) \quad \text { return } t_{i}\right\} \in \overline{\mathrm{M}^{\prime}} \\
& \operatorname{mbody}(\mathrm{m}, \mathrm{X}, \mathrm{C})=(\overline{\mathrm{x}}, \mathrm{t}) \\
& T T(\mathrm{X})=\text { expander } \mathrm{X} \text { of } \mathrm{T} \text { implement } \mathrm{S} \overline{\mathrm{I}}\{\overline{\mathrm{T}} \overline{\mathrm{f}}=\overline{\mathrm{V}} ; \overline{\mathrm{M}}\} \overline{\mathrm{O}} \\
& \text { of } \mathrm{C}\left\{\overline{\mathrm{M}^{\prime}}\right\} \in \overline{\mathrm{O}} \quad \mathrm{m} \text { is not defined in } \overline{\mathrm{M}^{\prime}} \\
& T T(\mathrm{C})=\text { class } \mathrm{C} \text { extends } \mathrm{D} \cdots \\
& \operatorname{mbody}(\mathrm{m}, \mathrm{X}, \mathrm{C})=\operatorname{mbody}(\mathrm{m}, \mathrm{X}, \mathrm{D}) \\
& T T(\mathrm{X})=\text { expander } \mathrm{X} \text { of } \mathrm{T} \text { implements } \overline{\mathrm{I}}\{\overline{\mathrm{T}} \overline{\mathrm{f}}=\overline{\mathrm{V}} ; \overline{\mathrm{M}}\} \overline{\mathrm{O}} \\
& C \text { is not defined in } \overline{0} \\
& T T(\mathrm{C})=\text { class } \mathrm{C} \text { extends } \mathrm{D} \cdots \\
& \operatorname{mbody}(\mathrm{m}, \mathrm{X}, \mathrm{C})=\operatorname{mbody}(\mathrm{m}, \mathrm{X}, \mathrm{D}) \\
& T T(\mathrm{X})=\text { expander } \mathrm{X} \text { of } \mathrm{T} \text { implement } \mathrm{S} \overline{\mathrm{I}}\{\overline{\mathrm{T}} \overline{\mathrm{f}}=\overline{\mathrm{V}} ; \overline{\mathrm{M}}\} \overline{\mathrm{O}} \\
& \mathrm{U} m(\overline{\mathrm{U}} \overline{\mathrm{x}}) \quad \text { return } t ;\} \in \overline{\mathrm{M}} \\
& \operatorname{mbody}(\mathrm{m}, \mathrm{x}, \mathrm{object})=(\overline{\mathrm{x}}, \mathrm{t})
\end{aligned}
$$

Figure 15. Helper rules for FeJ evaluation.

$$
\begin{aligned}
& \frac{\Gamma \vdash t: \mathrm{T} \quad \text { ftype }(\mathrm{f}, \mathrm{T})=\mathrm{U}}{\Gamma \vdash t . \mathrm{f}: \mathrm{U}} \\
& \Gamma \vdash \mathrm{t}_{0}: \mathrm{T}_{0} \\
& \operatorname{mtype}\left(\mathrm{m}, \mathrm{T}_{0}\right)=\overline{\mathrm{T}} \rightarrow \mathrm{T} \\
& \frac{\Gamma \vdash \overline{\mathrm{t}}: \overline{\mathrm{S}} \overline{\mathrm{S}}<: \overline{\mathrm{T}}}{\Gamma \vdash \mathrm{t}_{0} \cdot \mathrm{m}(\overline{\mathrm{t}}): \mathrm{T}} \\
& T T(\mathrm{X})=\text { expander } \mathrm{X} \text { of } \mathrm{T} \text { implements } \overline{\mathrm{I}}\{\ldots\} \overline{\mathrm{O}} \\
& \Gamma \vdash t: \mathrm{U} \quad \mathrm{U}<: \mathrm{T} \\
& \Gamma \vdash t \text { with } \mathrm{X}: \mathrm{U}^{\mathrm{X}} \\
& \frac{\Gamma \vdash t: T^{X}}{\Gamma \vdash \text { peel } t: T}
\end{aligned}
$$

Figure 16. FeJ typechecking for terms.

$X$. There are four cases. If $X$ has an overriding expander for $C$ containing a method $\mathrm{m}$, then that method's body is returned. If $\mathrm{X}$ either has an overriding expander for $C$ but that expander does not override $\mathrm{m}$, or if $\mathrm{X}$ has no overriding expander for $\mathrm{C}$, then we recursively search for an overriding expander for C's direct superclass D. Finally, if $\mathrm{C}=\mathrm{Object}$, then the body of the m method in the top expander is returned. Although object may not be a subtype of the expanded type, static typechecking ensures that mbody is always used in a type-correct manner.

Given this mbody function, the third rule in Figure 14 looks up the appropriate method body in X for the unexpanded object's runtime class $C$. The next rule handles the case when the expanded value itself has the form $\mathrm{v}^{\prime}$ with $\mathrm{X}^{\prime}$. In that case, the top expander's method is always invoked, without considering overriding expanders. This semantics makes sense since the run-time type of the expanded value has the form $\mathrm{T}^{\mathrm{X}^{\prime}}$, and such a type cannot be a subtype of any class type according to our subtype relation, so we are guaranteed that no overriding expanders are applicable. The semantics is encoded by invoking mbody with object as the third argument. Finally, if the expander $\mathrm{X}$ does not define $\mathrm{m}$, then lookup proceeds in the unexpanded object.

The rest of the rules in Figure 14 define the semantics of with and peel, which are straightforward.

\subsection{Static Semantics}

The rules for typechecking terms are presented in Figure 16, and helper functions are defined in Figure 17. Again, only the rules related to expanders are shown. A field access is type-correct if an appropriate field can be found for the type of the receiver. For a receiver of type $U^{X}$, the declaration of $X$ is searched for a field of the appropriate name. If the field is not found, the type $U$ is searched for a definition of the field. In this way, expanded objects are allowed to access fields of the unexpanded object (if they are not shadowed by the expander). Typechecking of method invocation proceeds analogously. The type rule for an expression $t$ with $X$ ensures that the type of $t$ is a subtype of the type expanded by $X$. The type rule for peel is straightforward.

Finally, Figure 18 contains the rules for typechecking expander declarations. We assume that the types being expanded in a given expander declaration are all pairwise distinct. Each overriding expander is required to be for a class that is a subtype of the type expanded by the top expander. The third rule describes how meth- 
ftype $(\mathrm{f}, \mathrm{T})=\mathrm{U}$

$$
\begin{gathered}
\frac{\operatorname{fields}(\mathrm{X})=\overline{\mathrm{T}} \overline{\mathrm{f}}=\overline{\mathrm{V}}}{\operatorname{ftype}\left(\mathrm{f}_{i}, \mathrm{U}^{\mathrm{X}}\right)=\mathrm{T}_{i}} \\
\frac{\operatorname{fields}(\mathrm{X})=\overline{\mathrm{T}} \quad \overline{\mathrm{g}}=\overline{\mathrm{v}} \quad \mathrm{f}_{i} \notin \overline{\mathrm{g}}}{\operatorname{ftype}\left(\mathrm{f}_{i}, \mathrm{U}^{\mathrm{X}}\right)=\operatorname{ftype}\left(\mathrm{f}_{i}, \mathrm{U}\right)}
\end{gathered}
$$

$\operatorname{mtype}(\mathrm{m}, \mathrm{T})=\overline{\mathrm{T}} \rightarrow \mathrm{T}$

$T T(\mathrm{X})=$ expander $\mathrm{X}$ of $\mathrm{T}$ implement $\overline{\mathrm{I}}\{\overline{\mathrm{T}} \overline{\mathrm{f}}=\overline{\mathrm{V}} ; \overline{\mathrm{M}}\} \overline{\mathrm{O}}$ $\mathrm{U} m(\overline{\mathrm{U}} \overline{\mathrm{x}}) \quad\{$ return $t ;\} \in \overline{\mathrm{M}}$

$\frac{\operatorname{mtype}\left(\mathrm{m}, \mathrm{S}^{\mathrm{X}}\right)=\overline{\mathrm{U}} \rightarrow \mathrm{U}}{\operatorname{TT}(\mathrm{X})=\text { expander } \mathrm{X} \text { of } \mathrm{T} \text { implements } \overline{\mathrm{I}}\{\overline{\mathrm{T}} \overline{\mathrm{f}}=\overline{\mathrm{V}} ; \overline{\mathrm{M}}\} \overline{\mathrm{O}}}$

Figure 17. Helper rules for term typechecking.

ods are typechecked. The method body is typechecked under the assumption that the receiver this has the expanded type. The OverrideOK judgment is used for typechecking methods in overriding expanders. In addition to the ordinary rules for typechecking methods, this judgment requires that a method of the same type signature appear in the top expander. That check is accomplished via the override helper function. Finally, the reallyImplements function ensures that each expander truly meets its declared interfaces.

A key property of this type system is modularity. Each class, interface, and expander is typechecked using only knowledge of its own declaration and the declarations of other types that it directly references. This means, for example, that an expander is typechecked without knowledge of what other expanders exist for the type being expanded, and without knowledge of all subtypes of the type being expanded.

\subsection{Type Soundness}

We have proven a type soundness theorem for FeJ using the standard "progress and preservation" style [34].

Theorem 4.1. (Progress) If $\bullet \vdash t: T$, then either $t$ is a value, $t$ contains a subexpression of the form (U) (v) where $\bullet \vdash \mathrm{v}: \mathrm{S}$ and $\mathrm{S} \not \mathrm{U}, \mathrm{U}$, or there exists some term $\mathrm{s}$ such that $\mathrm{t} \longrightarrow \mathrm{s}$.

THEOREM 4.2. (Type Preservation) If $\Gamma \vdash t: T$ and $t \longrightarrow s$, then there exists some type $\mathrm{S}$ such that $\Gamma \vdash \mathrm{s}: \mathrm{S}$ and $\mathrm{S}<: \mathrm{T}$.

The full proofs of these theorems are available in our companion technical report [33]. Together the theorems imply that well-typed FeJ programs cannot incur a type error at run time. This means that FeJ's modular type system is sufficient to guarantee the absence of the kinds of type errors that are caused by accidental overriding, as described in Section 3.3.

\section{Compilation}

The eJava compiler is built on top of the Polyglot extensible compiler framework for Java [22]. Like other Polyglot-based language implementations, our compiler translates eJava programs into equivalent Java programs, which can then be compiled with a standard Java compiler and run on a standard Java virtual machine

$$
\begin{gathered}
\bullet \vdash \overline{\mathrm{V}}: \overline{\mathrm{S}} \quad \overline{\mathrm{S}}<: \overline{\mathrm{T}} \\
\overline{\mathrm{M}} \mathrm{OK} \text { in } \mathrm{X}, \mathrm{T} \quad \overline{\mathrm{O}} \mathrm{OK} \text { in } \mathrm{X} \\
\text { reallyImplements }(\mathrm{T} \mathrm{X}, \overline{\mathrm{I}}) \\
\hline \text { expander } \mathrm{X} \text { of } \mathrm{T} \text { implements } \overline{\mathrm{I}}\{\overline{\mathrm{T}} \overline{\mathrm{f}}=\overline{\mathrm{V}} ; \overline{\mathrm{M}}\} \overline{\mathrm{O}} \mathrm{OK}
\end{gathered}
$$

$\mathrm{O} O \mathrm{OK}$ in $\mathrm{X}$

$$
\begin{aligned}
& T T(\mathrm{X})=\text { expander } \mathrm{X} \text { of } \mathrm{T} \text { implements } \overline{\mathrm{I}}\{\ldots\} \overline{\mathrm{O}} \\
& \mathrm{C}<: \mathrm{T} \quad \overline{\mathrm{M}} \text { OverrideOK in } \mathrm{X}, \mathrm{C} \\
& \text { of } \mathrm{C}\{\overline{\mathrm{M}}\} \mathrm{OK} \text { in } \mathrm{X}
\end{aligned}
$$

$\mathrm{M} \mathrm{OK}$ in $\mathrm{X}, \mathrm{T}$

$$
\frac{\overline{\mathrm{x}}: \overline{\mathrm{T}}, \text { this }: \mathrm{T}^{\mathrm{X}} \vdash \mathrm{t}_{0}: \mathrm{U}_{0} \quad \mathrm{U}_{0}<: \mathrm{T}_{0}}{\mathrm{~T}_{0} \mathrm{~m}(\overline{\mathrm{T}} \overline{\mathrm{x}})\left\{\text { return } \mathrm{t}_{0} ;\right\} \text { OK in } \mathrm{X}, \mathrm{T}}
$$

M OverrideOK in $\mathrm{X}, \mathrm{C}$

$$
\begin{gathered}
\operatorname{averride}\left(\mathrm{m}, \mathrm{X}, \overline{\mathrm{T}} \rightarrow \mathrm{T}_{0}\right) \\
\frac{\mathrm{T}_{0} \mathrm{~m}(\overline{\mathrm{T}} \overline{\mathrm{x}})\left\{\text { return } t_{0} ;\right\} \text { OK in } \mathrm{X}, \mathrm{C}}{\mathrm{T}_{0} \mathrm{~m}(\overline{\mathrm{T}} \overline{\mathrm{x}})\left\{\text { return } t_{0} ;\right\} \text { OverrideOK in } \mathrm{X}, \mathrm{C}}
\end{gathered}
$$

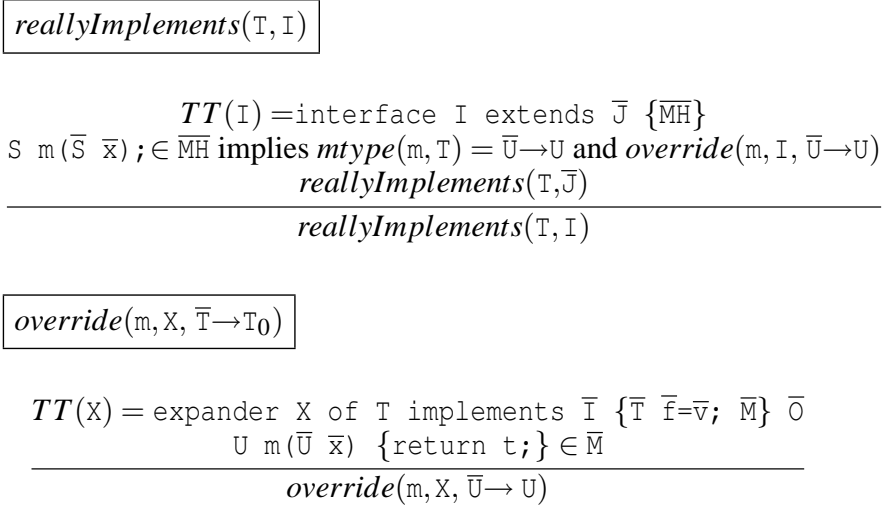

Figure 18. Typechecking for expander declarations.

(JVM). ${ }^{2}$ Polyglot compiles Java 1.4, so our eJava compiler inherits this limitation, for example lacking support for generics. We expect the issues for generic expanders to be analogous to those for classes, and we plan to pursue such an extension in the future.

The eJava compiler translates each expander family to a single Java class with the same name. Figure 19 shows the code generated for the EX expander from Figure 4. We refer to that example throughout this section.

\subsection{Wrapper Classes}

The functionality provided by an expander family is implemented in a set of wrapper classes, one for each expander in the family. In our example, the wrapper classes EX\$Expr, EX\$Value, and

\footnotetext{
${ }^{2}$ We have not yet given serious consideration to the option of adding support for expanders to the JVM, although we suspect this would allow for a much more efficient implementation.
} 


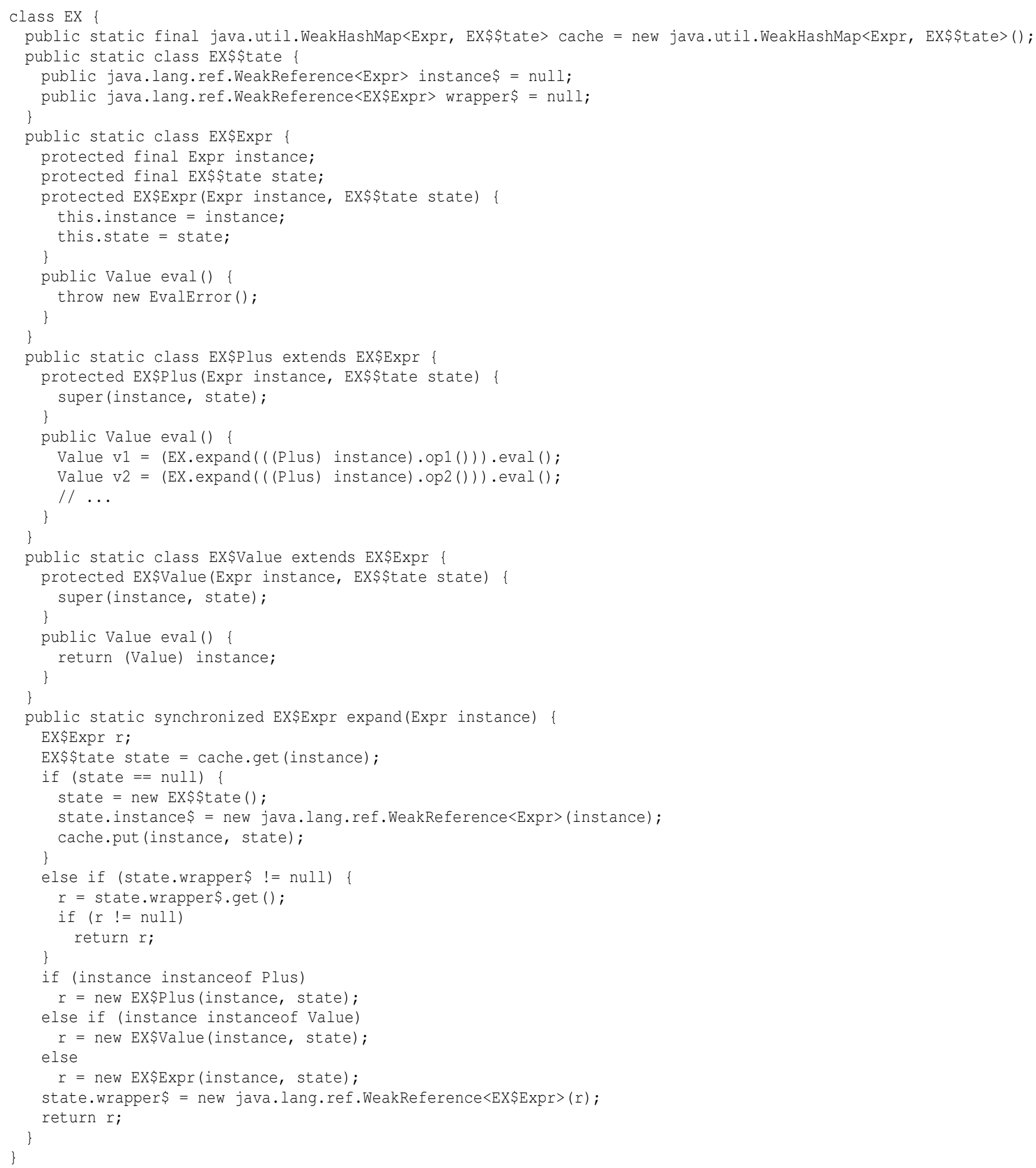

Figure 19. Java translation of the EX expander from Figure 4. 
EX\$Plus are generated for the expanders of EX whose associated classes are Expr, Value, and Plus, respectively.

Each wrapper class holds a reference to the original (unexpanded) object. This reference is used in the implementation of the methods of the expander. For instance, EX\$Plus's eval method uses it to call the op1 and op2 methods of its associated object. Note that the hierarchy of the wrapper classes mirrors the hierarchy of the types for which they were created. Consequently, in any given family, expanders of more specific types inherit-and may override, when desired-methods from expanders of less specific types.

\subsection{The expand Method}

Wrapper objects are created by the expander's expand method, which takes an unexpanded object and performs instanceof tests to determine the best (i.e., most specific) wrapper class to use for that object. The first part of the expand method in Figure 19 is used to properly handle fields in expanders; it is discussed in Section 5.4.

Clients of an expander call its expand method in order to access functionality it provides. For example, in Figure 5 the call e.eval () is translated to (EX.expand (e)) .eval ().

\subsection{Interface-Implementing Expanders}

Expander families that implement one or more interfaces, such as Figure 6's PX, are also translated as described above. Additionally, the wrapper class generated for the top expander's associated type is declared to implement those interfaces. This enables the client to use the expanded object (returned by expand) in contexts where any of the interfaces is expected.

An expander can employ methods of the type being expanded in order to meet new interfaces. To implement this functionality, we generate forwarding methods in the wrapper class for the top expander, which simply invoke the corresponding methods on the unexpanded object. All interfaces in Java implicitly include signatures for the public methods of java.lang.0bject, like equals, toString, and hashCode, so forwarding methods for them are also created (except in cases where the method is explicitly shadowed by the expander).

These forwarding methods are only ever used when an expanded object is viewed through one of the interfaces that the expander meets. They are not necessary in all other situations, because in those cases eJava's semantics will cause the appropriate methods to be called directly on the unexpanded method. For example, if e has static type Expr then e.tostring() will always invoke the tostring () method defined for the run-time class of e, without considering any of the expanders in scope.

When an expanded object is viewed through one of the interfaces that the expander meets, the forwarding methods enable the expanded object to "pretend" to be the original object as much as possible. However, one well-known limitation of wrappers is that they do not preserve object identity. Therefore, code that employs $==$ or instanceof on an expression of an interface type can get incorrect results if that expression evaluates to an expanded object. This limitation is an artifact of our modular compilation strategy and is also a limitation of the adapter design pattern [15].

An alternative compilation strategy that nonmodularly modifies a class in place would not suffer from this problem, but it would suffer from other problems. For example, it would be impossible to adapt a class to support two incompatible interfaces (e.g., interfaces that define the same method but with a different return type) in two different expanders. Both the AspectJ and Classboxes implementation strategies suffer from these kinds of problems.

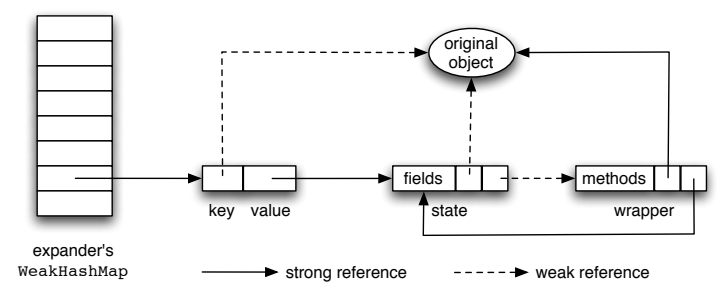

Figure 20. Expander state and wrapper objects.

\subsection{Handling State in Expanders}

One straightforward way to represent the fields of an expander family in our implementation scheme would be to declare those fields in the wrapper class generated for that family's top expander. Wrapper objects would additionally be cached for use by the expand method, in order to give clients of the same object a consistent view of its new fields' values. Unfortunately, this simple approach interacts poorly with Java's garbage collection: expand's cache would hold a reference to every object ever adapted by the expander, preventing those objects from ever being garbage collected.

Java has a notion of weak references, which, unlike the usual strong references, do not prevent their referents from being collected. One possible solution to the above problem is for the cache to hold weak references to unexpanded objects. Although this approach does not result in memory leaks, it can cause dangling pointer errors: it is possible for the original object to be garbage collected while one or more clients still hold a reference to the associated wrapper object.

Our implementation scheme, outlined in Figure 20, avoids both of these problems. The eJava compiler generates a state class for each expander, and each wrapper object maintains a reference to an associated state object. The EX\$ $\$$ tate class in Figure 19 is the state class generated for the EX expander. If EX declared a field $f$, it would be declared in this state class. Client code referring to e.f, where e has type Expr, would be translated to EX. expand (e) . state.f.

Like wrappers, state objects are instantiated when an object is expanded. As in the approach discussed above, the expand method uses a WeakHashMap, a hashtable implementation that holds weak references to the objects it uses as keys, to cache the state objects for each object expanded. An important property of WeakHashMap is that values associated with keys that are garbage collected are automatically removed. This feature ensures that state objects whose associated unexpanded objects are no longer accessible are properly disposed of, avoiding memory leaks.

For performance reasons, the state object contains a wrapper\$ field, which is used to cache the associated wrapper object. This allows the implementation to avoid instantiating a new wrapper object every time a client accesses functionality by an expander on the same object.

To solve the dangling pointer problem, the wrappers generated by our translation hold strong references to their original objects. This ensures that the original object will not be garbage collected as long as there is at least one client who still holds a reference to the wrapper.

Should it ever be the case that no client holds a reference to the original object or to its wrapper, the wrapper's strong reference to the object will not keep the object from being garbage collected. In such scenarios, the only object in the system which has a reference to the wrapper is the state object. And because this reference is weak, it does not prevent the wrapper-and consequently the original object-from being collected.

Finally, suppose one or more clients have strong references to the original object, but no client has a strong references to the 


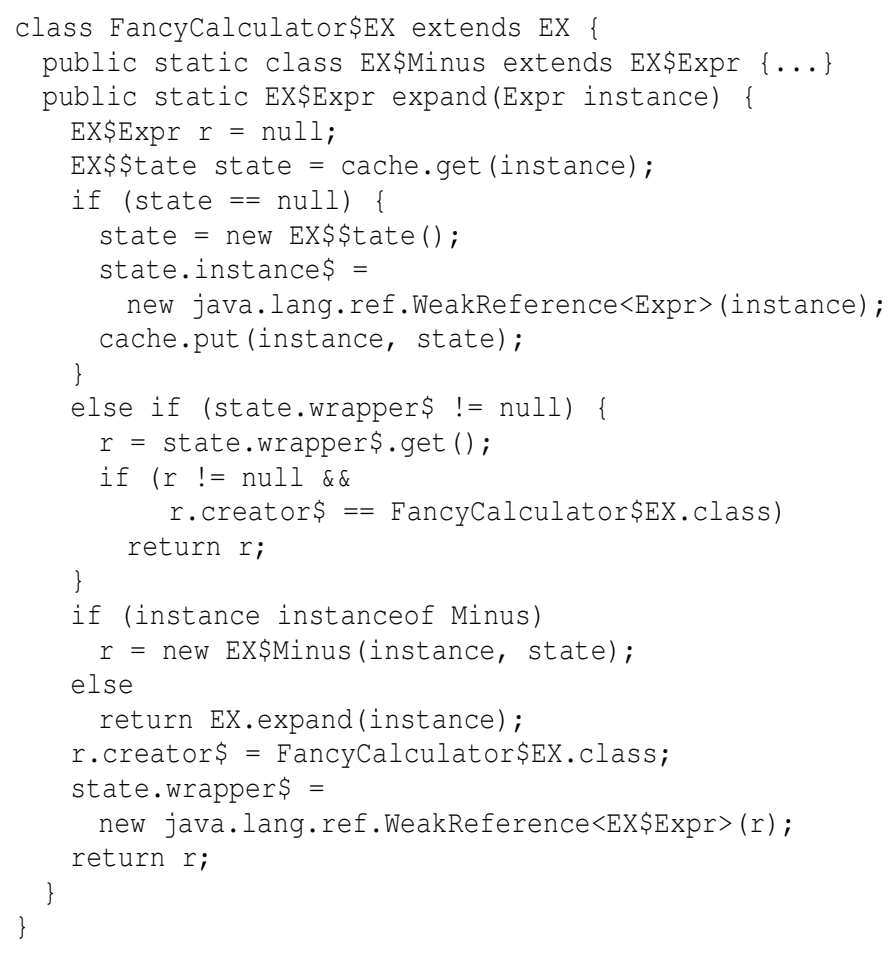

Figure 21. The translation of the local overriding expander in Figure 11.

associated wrapper. Here, it is possible that the wrapper will be garbage collected. Fortunately, because our wrappers are stateless, they can be instantiated as often as needed. This is done in the expander's expand method (see Figure 19).

\subsection{The expand Method, Revisited}

Now that most of eJava's implementation details have been revealed, we are able to provide a more detailed description of the actions carried out by the expand method.

Step 1: expand searches the expander's cache for the state object associated with the object to be expanded. If the search succeeds and the state object's wrapper exists, expand returns the wrapper object. If the search fails, a new state object whose instance $\$$ field references the instance to be expanded is created and stored in the cache.

Step 2: If we get to this point, we've got a state object, but no wrapper. expand then creates the most-specific wrapper based on the dynamic type of the object to be expanded. A weak reference to this object is stored in the state object, and the wrapper is returned to the caller.

\subsection{Local Overriding Expanders}

As discussed in section 2.5, clients may augment an expander family with any number of local overriding expanders. Our compilation scheme supports this ability by generating a subclass of the original expander's implementation class. For example, Figure 21 shows the code generated for the local overriding expander shown in Figure 11. The new class contains the appropriate wrapper classes, which are declared to be subclasses of the wrapper classes of the original expander. The state class created for the original expander is inherited for use by the local overriding expander. When creating new wrapper objects, the new expander class's expand method

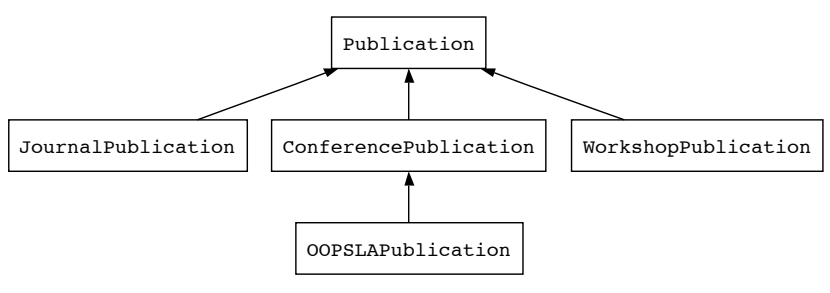

Figure 22. The hierarchy of publication classes.

uses instanceof tests to determine whether one of its refinements should be used for the instance being expanded. If this is not the case, it forwards the call to the expand method of its superclass. The uses of locally augmented expanders are compiled as calls to the new expander class's expand method (and not that of the original expander).

To adhere to eJava's semantics as described earlier, wrappers created by local expanders should never be returned by expand in contexts other than their own. Similarly, a wrapper created for a particular instance by the original expander should not be used in a context where a local overriding expander exists for the dynamic type of that instance. In order to prevent these scenarios, an expander's expand method never returns a cached wrapper object that was created by a different expand method. Figure 21 shows how FancyCalculator $\$ E X$ 's expand method enforces this restriction with the help of a creator $\$$ field in each wrapper class. Since any expander family may be augmented by clients through local overriding expanders, this check is performed by the expand method of every expander, regardless of whether or not the the existence of local overriding expanders is known at compile time (for clarity, this mechanism was omitted from the implementation of EX shown in Figure 19).

\section{Experience}

This section describes two experiments we performed to gauge the practical utility of eJava's expanders. First, we considered a scenario in which a client must adapt existing objects in order to display them in a tree structure using Swing's JTree class. We implemented solutions in both Java and eJava to compare the benefits and limitations of each approach. Second, we performed an exploratory study of the Eclipse IDE framework [8]. The goal of the study was to understand the various extensibility idioms used in Eclipse and the extent to which expanders allow these idioms to be expressed more naturally and/or reliably.

\subsection{Making Objects "Swing”}

Consider a database of objects that represent various academic publications, with each publication linking to the publications it references. The publications in this database are represented by the Publication hierarchy, shown in Figure 22. Since different clients of this database are likely to want to display publications in different ways, the classes in the Publication hierarchy do not provide any displaying capabilities on their own; this functionality must be implemented by each client that requires it. Further, because the publication objects come from a database (and are not instantiated by clients themselves), this functionality must be added externally.

This case study addresses how one client might display the objects in this database graphically as a tree-with each node representing a publication and subnodes representing the publication's references-using Swing's JTree class. Figure 23 shows a screenshot of the client application. Note that each kind of publication is displayed in its own style, consisting of a particular icon and text format. 


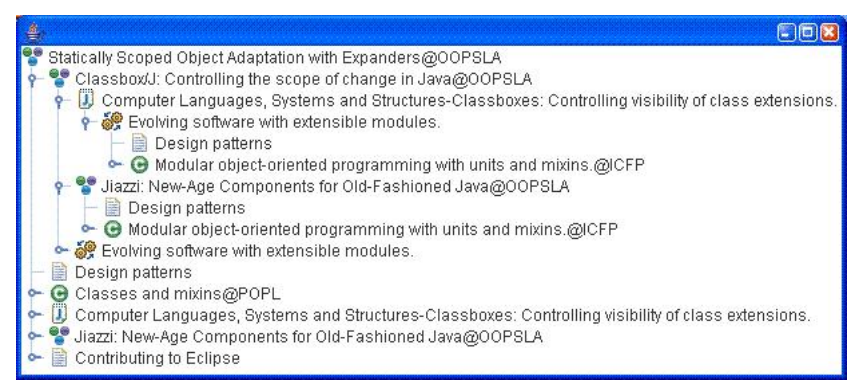

Figure 23. The client application.

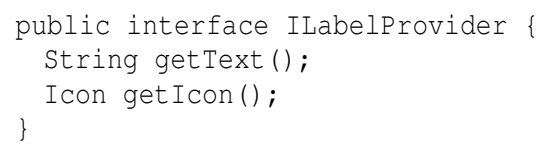

Figure 24. The ILabelProvider interface.

To display objects in a JTree, clients must provide an implementation of Java's TreeModel interface, which provides a data model for the tree. As a convenience, the Swing library provides a default implementation of this interface, DefaultTreeModel. However, DefaultTreeModel requires the objects in the tree to implement the TreeNode interface. Therefore, in order to avoid duplicating the code of DefaultTreeModel, the publication objects must be adapted to meet the TreeNode interface.

To customize the way objects in a JTree are displayed, the client must also implement the TreeCellRenderer interface. This interface has a single method, get TreeCellRendererComponent, which takes an Object and returns the graphical Component to be used for displaying that object. The simplicity of this interface often complicates the implementation of the get TreeCellRendererComponent method, which must perform instanceof tests and type casts if different kinds of objects should be displayed differently. Therefore, many Swing applications define their own interface for rendering, which is implemented by the object passed to getTreeCellRendererComponent. The implementation of that method can then delegate most of the rendering work to the given object by calling appropriate methods from this new interface. In our application, we require the objects in the tree to support the ILabelProvider interface, shown in Figure 24, which allows each kind of publication to provide its own text and icon for display. Therefore, Publication objects must also be adapted to meet the ILabelProvider interface.

\subsubsection{The eJava Version}

Our eJava implementation uses expanders to adapt the various types of publications to meet the TreeNode and ILabelProvider interfaces. Figure 25 shows some of the code in the top expander (i.e., the one for Publication). The first several methods in the expander implement the TreeNode interface. Note the use of the with operator to explicitly expand $\mathrm{p}$ (an instance of Publication) in the children method. This is necessary because we intend to store the expanded versions of those objects in a Vector. Since Vector's add method takes an argument of type Object, no adaptation is necessary, and therefore $\mathrm{p}$ is not expanded automatically. Java 1.5's generics would allow us to type children as Vector<TreeNode>, which would then result in the implicit adaptation of $p$, but as mentioned earlier, the eJava compiler does not currently support generics.

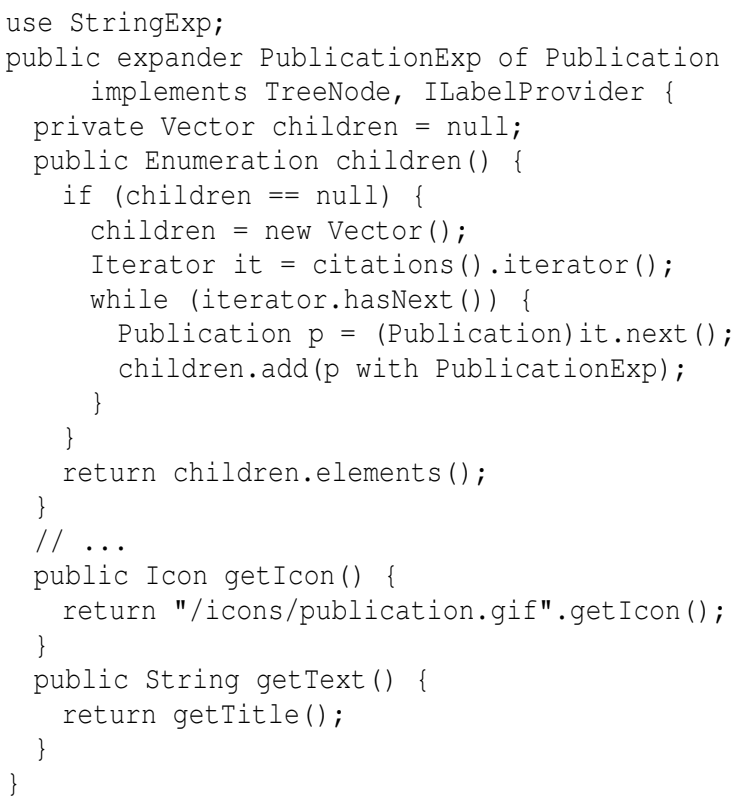

Figure 25. The PublicationExp expander.

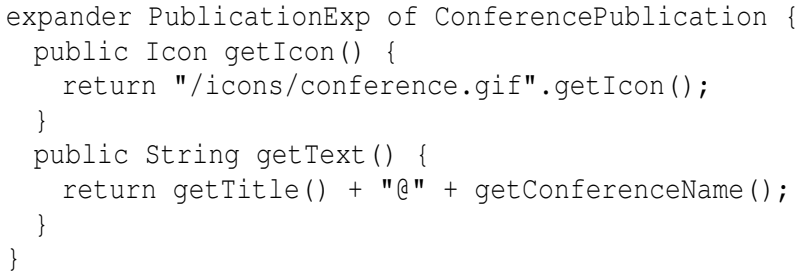

Figure 26. An overriding expander.

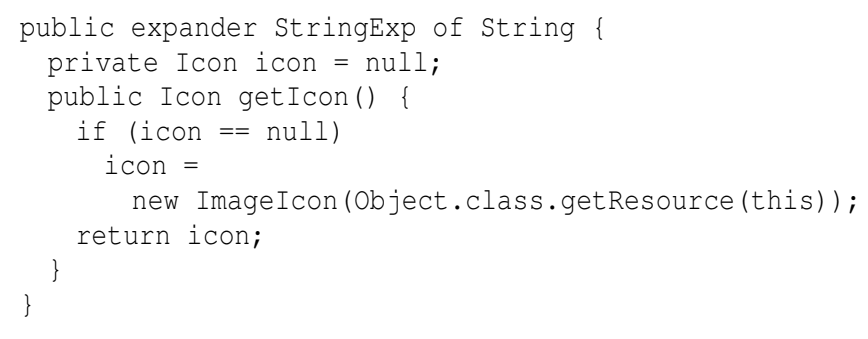

Figure 27. Retrieving icons from a file.

The expander in Figure 25 provides a default implementation of ILabelProvider for instances of Publication. Overriding expanders customize the presentation of each kind of publication by providing their own implementation of the get Text and get Icon methods. For example, Figure 26 shows the overriding expander for ConferencePublication.

We use an expander for String to encapsulate the logic for loading icons from files, as shown in Figure 27. The expander also caches the icon in its icon field. Since in Java identical string literals are represented by the same underlying String object, our application will not load an icon more than once from the same file. 


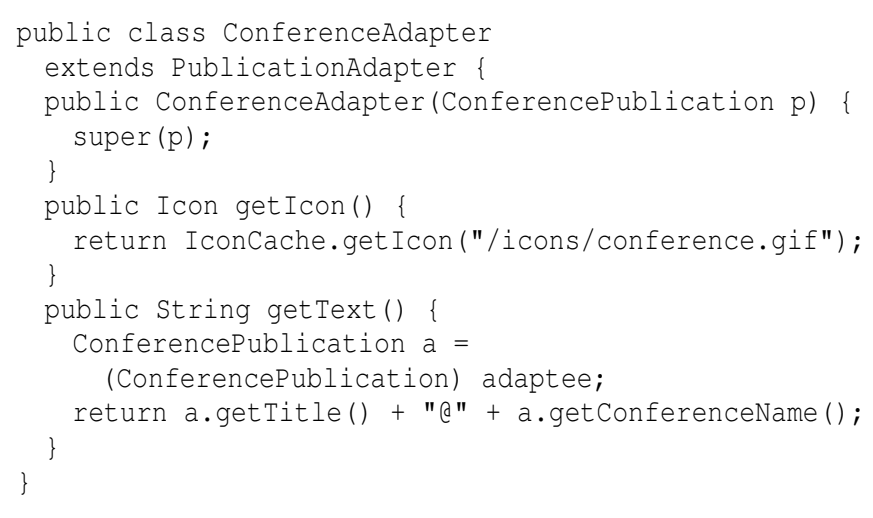

Figure 28. An adapter for ConferencePublication.

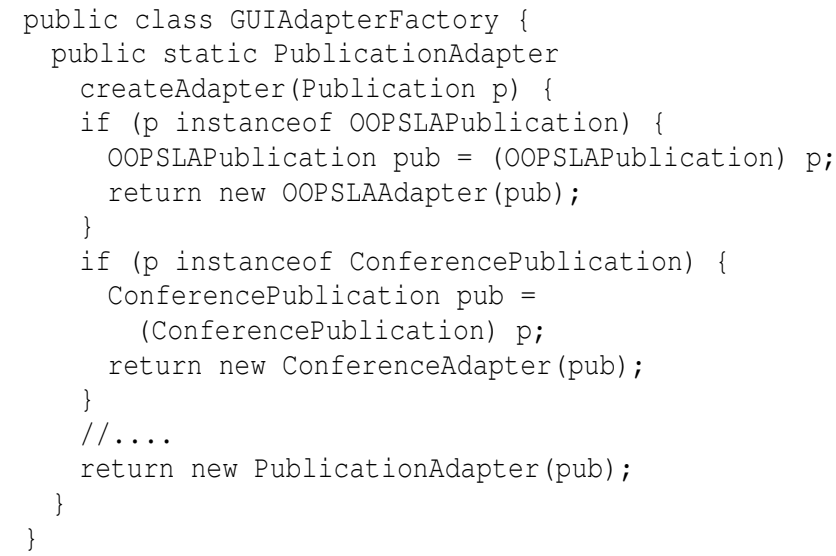

Figure 29. The adapter factory.

\subsubsection{The Java Version}

Our Java implementation employs the adapter design pattern [15]. To encode special rendering behavior for each subclass of Publication, we create a hierarchy of adapter classes that mirrors the publication hierarchy. The root of the adapter hierarchy is PublicationAdapter, which looks similar to the PublicationExp expander, except that it maintains an explicit field adaptee of type Publication to access the underlying publication object. Similarly, the other classes in the hierarchy of adapters correspond to the overriding expanders of the eJava implementation. For example, the ConferenceAdapter, which is the analogue of the overriding expander for ConferencePublication (Figure 26) is shown in Figure 28. Since the adapter makes use of specific features of ConferencePublication, like getConferenceName, the adaptee field must be downcast appropriately.

Our Java implementation is required to perform instanceof tests in order to determine the correct adapter class for a publication object. The createAdapter method in Figure 29 implements this functionality. The instanceof tests must be performed in most- to least-specific order to ensure that each object is adapted properly. It is possible to do away with the adapter hierarchy and instead use a single adapter class for all publications. However, in that case this sequence of instanceof tests would be required in the implementation of every method that has specialized behavior for
Publication subclasses, instead of occurring only once at the point of adaptation.

\subsubsection{Comparison}

This case study exposes several advantages of expanders over standard adapters in Java. First, the eJava version is more easily extensible. For example, consider the task of adding special support for TOPLASPublication, which is a subclass of JournalPublication. In the eJava version, one would simply add a new overriding expander for PublicationExp, either in the same source file as the original expanders or via local expander overriding. In the Java version, one would analogously need to add a new adapter class, TOPLASAdapter, which inherits from JournalAdapter. However, it would also be necessary to update or create a subclass of the GUIAdapterFactory (from Figure 29), in order to add a new case to createAdapter's cascading if statement that appropriately adapts a TOPLASPublication. Further, care must be taken to place this case in the appropriate order with respect to the existing cases.

The eJava version is also less error prone than the Java version. The latter version requires instanceof tests and associated downcasts in GUIAdapterFactory, in order to create the adapters, and additional downcasts within the adapters to narrow the type of the adaptee field, as shown earlier. In contrast, the eJava version requires none of these instanceof tests or downcasts. Instead, eJava's modular type system ensures type correctness statically. In total, the Java version of our simple application requires four instance of tests and eight downcasts. The eJava version contains no instanceof tests and three downcasts. Two of these downcasts would have been avoided with the use of generics, and the third occurs in the implementation of TreeCellRenderer, in order to narrow the Object argument of get TreeCellRendererComponent to ILabelProvider; it is also present in the Java version.

Finally, the eJava version has a performance advantage over the Java version. As described in Section 5, the instances of wrapper classes used in the implementation of an expander are cached for each expanded object. Therefore, the tree display in our application will create exactly one wrapper object per publication object displayed, even if the publication appears multiple times in the tree. In the Java version, each node in the tree has a separate adapter instance. Similarly, we remarked earlier that the StringExp expander naturally ensures that each icon is loaded from a file at most once. In the Java version, this optimization had to be implemented with an explicit icon cache.

\subsection{An Exploratory Study of Eclipse}

Eclipse is built with extensibility as a primary goal: the framework is structured as a small kernel, known as the Eclipse platform, and a collection of external plugins that provide the bulk of the system's functionality. As such, Eclipse represents a rich source for understanding the state of the art in Java-based extensibility idioms. In the rest of this section, we describe several of these idioms and their relationship to eJava's expanders.

\subsubsection{External Methods}

Eclipse uses two main approaches to add methods to existing classes from the outside. First, it provides two kinds of visitors: ASTVisitor, for traversing the AST representation of a program, and IResourceVisitor, for traversing trees of resources such as files, folders, and projects. These types, along with the appropriate "hooks" inside the classes representing AST nodes and resources, allow for easy extension of these hierarchies with new methods. However, only these two particular class hierarchies can be extended with new methods, and such extension is only possible because of advance planning by the Eclipse developers. Expanders, 


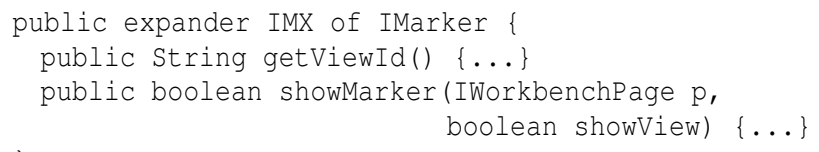

Figure 30. The IMarker expander in eJava.

on the other hand, allow any existing class hierarchy to be easily augmented with new methods, without the need to plan ahead for such extension. Further, they do not suffer from the standard problems of visitors, mentioned in Section 2.1. For example, each expander method can have its own argument and result types.

However, visitors do have one important advantage over expanders. Since each external method is reified as a visitor class, it is easy for external methods to inherit code from one another. A common usage of this ability is to define a visitor that does nothing except recursively visit the children of each node in the given tree. A new visitor class can then subclass from that visitor and inherit the code for traversing the tree "for free," only overriding the particular methods that should perform some useful work. In contrast, method families in an expander are completely unrelated to one another, forcing each to re-implement the basic traversal behavior. We believe this limitation could be solved with a notion of expander inheritance. We are currently working on adding support for this feature to the eJava compiler using a compilation strategy similar to the one used to support local overriding expanders.

Second, Eclipse has several utility classes that use static methods to provide new functionality to existing types. For example, the MarkerViewUtil class contains two methods that manipulate instances of the IMarker interface, with the following signatures:

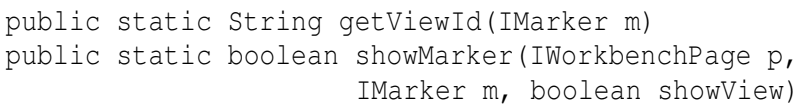

This approach to adding new methods to existing types has two main drawbacks. First, callers have to use a verbose and unnatural syntax to invoke such methods, for example MarkerViewUtil.getViewId (m), which clashes with the conceptual intent that the new methods are part of the existing IMarker type. Second, if a method needs to dispatch on the run-time class of the IMarker instance in order to determine how to behave, then the programmer must resort to error-prone instanceof tests and type casts, as shown in earlier sections.

Expanders naturally solve both of these problems. Figure 30 shows an expander for IMarker that adds the two methods described above. These new methods can be invoked using the ordinary calling syntax for methods of IMarker. Further, the implementer can provide overriding expanders in order to specify the behavior of each new method for particular classes that implement the IMarker interface. The eJava language then does the work of automatically dispatching to the appropriate implementation based on the run-time class of the receiver.

\subsubsection{Multiple Implementation Inheritance}

Eclipse employs a style in which clients only manipulate an abstraction through an interface, rather than a class. This style cleanly separates interface from implementation, ensuring that clients cannot depend on implementation details and allowing implementers to change the underlying implementations without affecting clients. To aid implementers, Eclipse often provides an abstract class that contains default implementations of a particular interface's methods. Implementers can then easily create a class meeting the desired

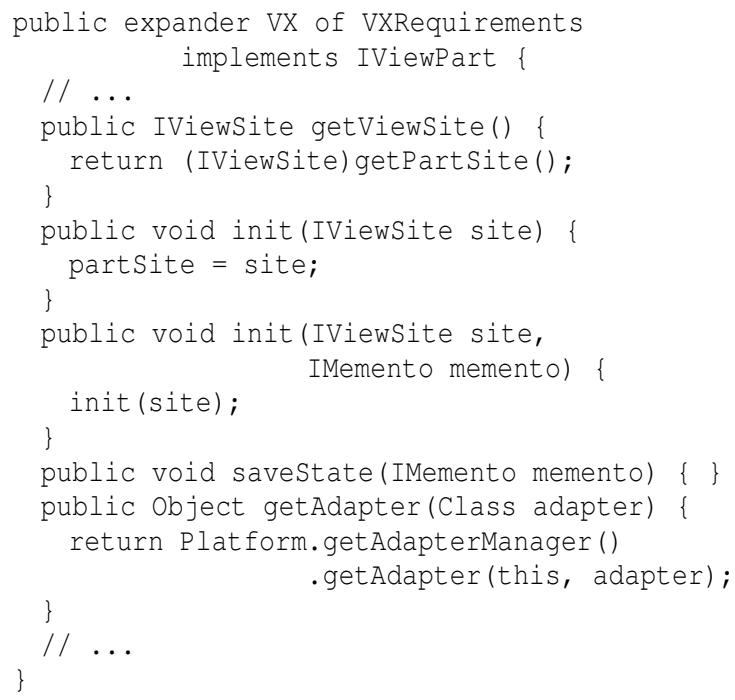

Figure 31. An expander replacing the ViewPart abstract class.

interface by inheriting from the associated abstract class and overriding methods as appropriate [14]. For example, Eclipse's concept of a view provides a visual representation of some content. A view must implement the IViewPart interface, which declares or inherits a total of 14 method signatures, and the abstract class ViewPart contains default implementations of 12 of these methods, requiring concrete subclasses to implement the other two.

Because Java does not support multiple inheritance, however, a class that subclasses ViewPart cannot inherit code from any other classes. This limitation can lead to a significant amount of code duplication. For example, suppose a class that subclasses ViewPart must meet another interface in addition to IViewPart. It is not possible for the class to inherit the default implementations of the other interface; instead, the implementer must explicitly copy these methods in the new class.

Expanders provide a natural solution to this problem. First we create an interface for the two methods that each concrete subclass of ViewPart is required to implement:

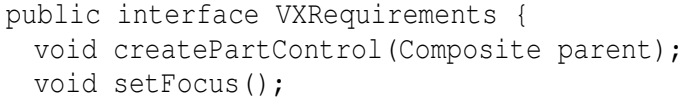

Then we create an expander that takes the place of the ViewPart class, providing the implementations of the other 12 methods required by IViewPart. This expander is shown in Figure 31. Any class that implements the VXRequirements interface can now be "expanded" to meet the IViewPart interface and thereby "inherit" the default implementations of the associated methods. Other expanders can be used in a similar fashion to allow the class to easily meet other interfaces, without requiring code duplication. Further, the class can still inherit code from a superclass, as usual in Java.

\subsubsection{Adapters}

Eclipse includes an adapter framework that allows an object to be dynamically extended to meet a new type. A type declares its instances to be adaptable by implementing the IAdaptable interface. Clients of that type can implement an adapter factory which specifies how to adapt that type's instances to new types, and register this factory with a global registry. An adapter factory from Eclipse that 


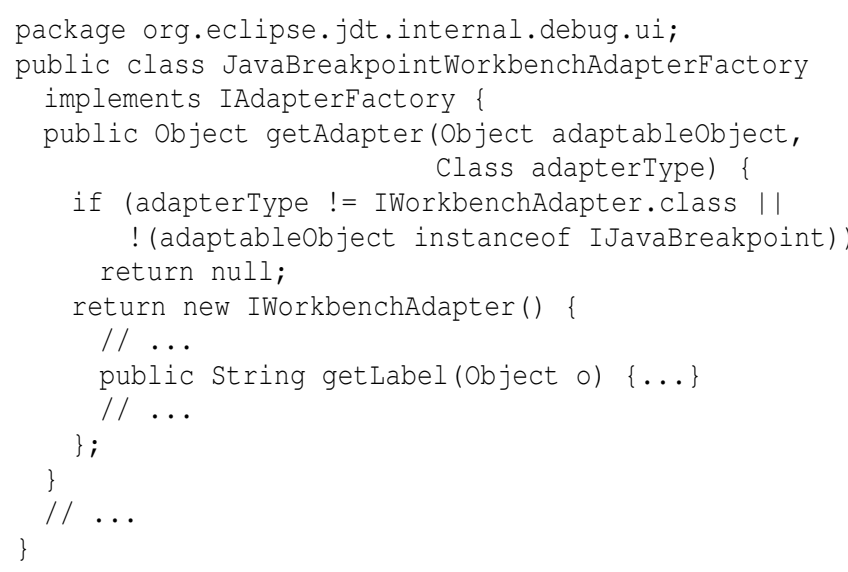

Figure 32. An example adapter factory from Eclipse.

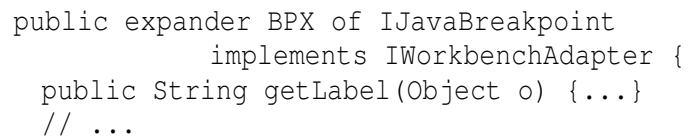

Figure 33. Adapting with an expander.

adapts instances of IJavaBreakpoint to IWorkbenchAdapter is shown in Figure 32. The adapter factory can then be invoked via the getAdapter method of the adaptee object, for example:

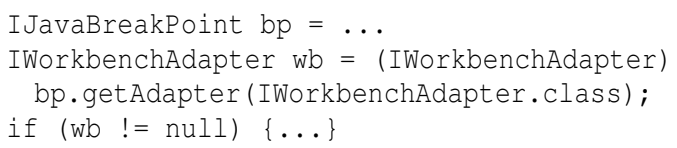

The adaptee object's getAdapter method typically forwards to the global registry, which uses the get Adapter method of each adapter factory to search for an appropriate adapter, returning null if one cannot be found.

With expanders, we can express both the adapter and its clients more naturally. Figure 33 provides an expander that adds the appropriate methods to an IJavaBreakpoint instance in order for it to meet the IWorkbenchAdapter interface. A client of an IJavaBreakpoint instance simply uses the expander BPX in order to implicitly adapt the instance to IWorkbenchAdapter, thereby allowing methods like getLabel to be called on that instance.

There are several interesting points of comparison between the two approaches. First, it is statically apparent in the declaration of an expander what type is being adapted and to what other type it is being adapted. This makes it easy for clients to understand the intended behavior of an expander. Similarly, it is statically visible through a compilation unit's use statements which expanders are being employed by any particular client. In contrast, Eclipse's adapter framework is inherently dynamic. The code of the getAdapter method in Figure 32 must be inspected to understand what types are involved in the adaptation. Further, it is impossible for a client to tell which adapter will be used when it calls getAdapter on an object. Of course, such dynamism can also be an advantage, by providing more expressiveness than is supported by expanders. For example, the getAdapter method in Figure 32 can easily perform the adaptation in one of several ways, depending on run-time conditions. Eclipse is structured around a dynamic plugin model, so it often requires such expressiveness.
Second, Eclipse's adapters are globally registered, which can cause problems. For example, if two plugins each register different adapter factories for the same source and target types, one of these factories will be shadowed, causing one plugin to use an adapter that may not be appropriate for its needs. Since expander usage is statically scoped, there can naturally exist multiple expanders for the same source and target types, and different clients can use different expanders without conflict.

Third, a type is only adaptable in Eclipse's framework if it is declared to meet the IAdaptable interface (and provides a getAdapter method). Therefore, the original implementer of a type must plan ahead for adaptation. Unfortunately, this advance planning often does not happen, as indicated by several requests in the Eclipse Bug System [9], shown in Table 1. Fourth, Eclipse's framework allows objects to be adapted to both new classes and new interfaces. In contrast, eJava's expanders allow a class to meet new superinterfaces but not new superclasses.

Finally, some Eclipse adapters are designed to be stateless: instead of storing the adaptee object as a field of the adapter object and delegating to this field as necessary, the adapter object's methods all explicitly accept an adaptee object as a parameter. The example in Figure 32 is in fact a stateless adapter. For example, if bp is an instance of IJavaBreakPoint and wb is the IWorkbenchAdapter object returned from the call bp.getAdapter(IWorkbenchAdapter.class), then the label text of the adapted object is accessed by the invocation wb. get Label (bp).

The stateless adapter design allows one adapter instance to be used across all objects that need to be adapted. In contrast, our compilation strategy for expanders, as discussed in Section 5, always creates one adapter object per adaptee object, thereby consuming more space. However, stateless adapters come at the cost of increased complexity, both for the adapter implementer and the adapter client. On the implementation side, the code for get Label must downcast the given Object to an IJavaBreakPoint before retrieving its label. On the client side, callers must explicitly manipulate both the adaptee object and its adapter.

\subsubsection{External State}

Eclipse allows plugins to add new state to existing objects that represent system resources via a notion of properties. A property is a name-value pair that can be added to an existing IResource instance. Properties are updated and accessed through two methods in the IResource interface:

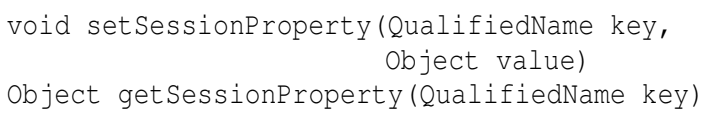

For example, Figure 34 shows how Eclipse's BuildManager class manipulates a property K_BUILD_LIST on instances of IProject.

The property mechanism is useful but has several drawbacks. First, it cannot be used to add new state to objects that are not resources. Second, properties are not statically typed. For instance, nothing prevents two calls to setSessionProperty from storing objects of different types to the same property. Since most callers of get SessionProperty downcast the resulting object to the expected type of the property, such type disagreement could lead to run-time errors like ClassCastExceptions. Third, each property of an object is globally accessible by all of the object's clients, which can lead to conflicts. For example, two clients could accidentally use the same QualifiedName to represent two distinct properties, causing unexpected interactions between these clients at run time.

Expanders allow state to be added to existing objects without suffering from any of these problems. For example, the following 
Table 1. A sample of the Eclipse Bug System's "should implement IAdaptable" bugs.

\begin{tabular}{|l|l|}
\hline Bug ID & Summary \\
\hline 12960 & All Update Core model objects must implement IAdaptable \\
109138 & IWorkingSet should extend IAdaptable \\
23032 & Make IEditorReference adaptable \\
22452 & Would like ITextViewer be a supported Adaptable for Abstract TextEditor [api] \\
80671 & proxies should be adaptable \\
\hline
\end{tabular}

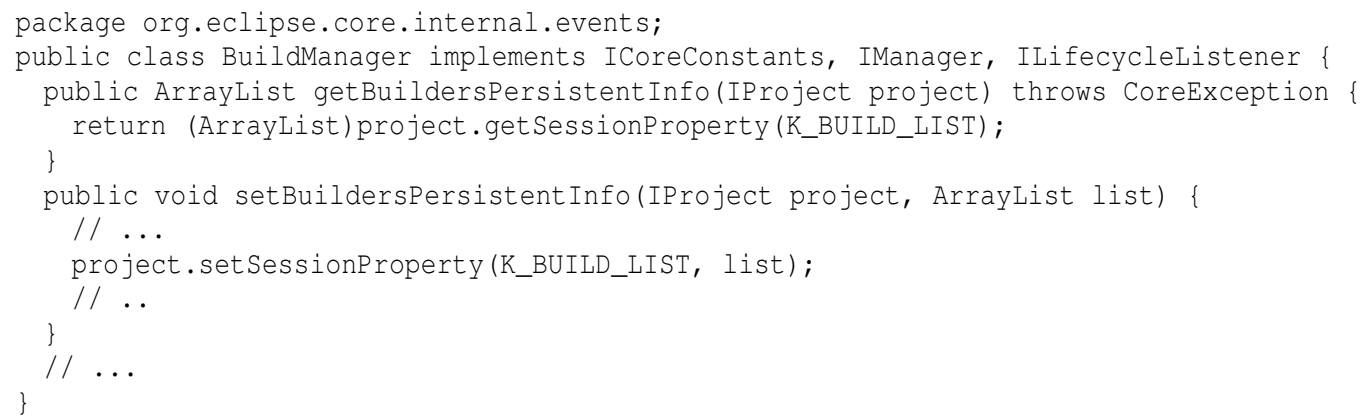

Figure 34. BuildManager in Java.

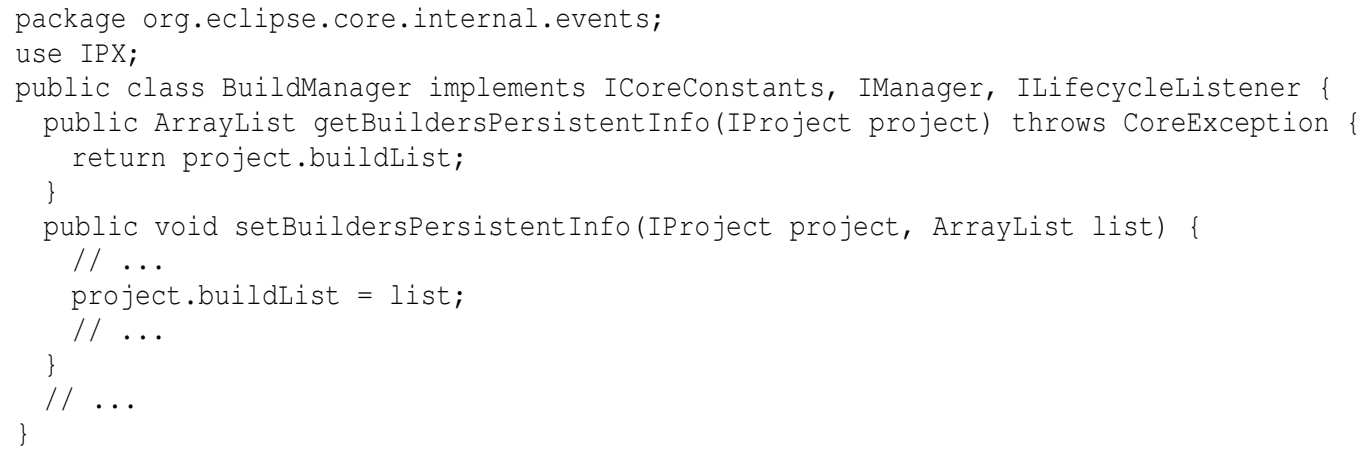

Figure 35. BuildManager in eJava.

expander augments IProject instances with a new field of type Arraylist:

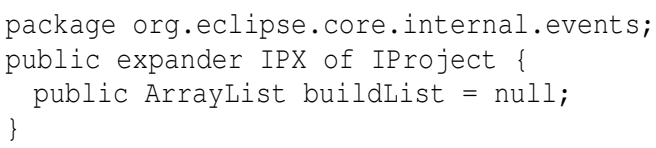

Clients like BuildManager can then use this expander and manipulate the new field as it would any other field, as shown in Figure 35. Accesses to the field are now statically typechecked, and different clients can use different expanders without any possibility of conflicts.

\section{Related Work}

Classboxes, a form of module for OO languages, are the closest language construct to expanders of which we are aware. Classboxes were originally developed in the context of Smalltalk [4] but were recently described in an extension to Java [3]. Classboxes allow existing classes to be refined to support new fields, methods, and superinterfaces, as in eJava. Classboxes also support the addition of new constructors and inner classes, as well as the overriding of existing methods of the class, all of which eJava does not support.
There are several important differences between expanders and classboxes. First, like expanders, the refinements in a classbox are only available where that classbox has been explicitly imported. While the visibility of a classbox is scoped, any class members added in a classbox are treated semantically as if they were defined in the original class declaration [2]. This semantics causes the kinds of accidental overriding problems described in Section 3.3, which elude modular detection. eJava's method-lookup semantics and modular type system prevent accidental overriding and the associated type safety problems.

Second, classboxes are implemented nonmodularly: all classboxes that refine a class must be available when that class is compiled, and these refinements are weaved into the class's declaration. At that point, regular Java typechecking and compilation are performed on the resulting class. This means that any errors in a classbox are not detected modularly. Further, while this implementation strategy avoids the problem of object identity for wrappers, it allows unrelated classboxes to clash with one another, preventing compilation. For example, if two classboxes define a method $\mathrm{m}$ for class $\mathrm{C}$, the methods are merged into a single m method that tests the current scope dynamically to determine what code should be executed. If these methods have different return types, the resulting 
Java code will not typecheck. While this problem could potentially be addressed by a form of name mangling, that strategy would not work if the methods are needed in order to implement particular interfaces. In contrast, because expanders are implemented modularly, two unrelated expanders cannot conflict with one another.

Finally, clients can only import a single classbox for a given class, while clients in eJava can use multiple expanders for the same class.

The inter-type declarations in Aspect J [19] support similar augmentations to a class as do classboxes. However, aspect usage is not scoped: an aspect's inter-type declarations are implicitly available to clients of the original classes. This semantics reflects the fact that aspects are often intended to update the behavior of existing classes in a manner that automatically updates all existing clients, in order to noninvasively evolve existing applications. While this behavior is quite powerful, it can also easily cause accidental relationships and conflicts among logically independent aspects and classes. Finally, AspectJ employs a similar compilation strategy as described above for classboxes and so suffers from similar problems.

The concept of subject-oriented programming [17] has goals that are very similar to our own. The basic idea is to allow different clients of a class to have different subjective views of that class's behaviors. Work on subject-oriented programming has explored mechanisms that allow programmers to specify rules for composing multiple "subjects," each of which encapsulates some of a class's behaviors [24]. Similarly, the follow-on work on $h y$ perslices $[30,25]$ allows programmers to explicitly compose separate hyperslices in flexible ways in order to produce the full class for use by an application. eJava does not support explicit composition of expanders, instead using ordinary static scoping to disambiguate among them. While less expressive, our approach allows expanders to be typechecked fully modularly. In contrast, conflicts among subjects or hyperslices can only be detected when they are composed to produce a particular full class, as with classboxes and aspects.

The Scala [27] programming language contains a construct called views, which are a form of language support for adaptation. Views are essentially programmer-defined functions from one type to another. However, the language infers where a view should be inserted, in order to allow a value of one type to be treated as having a different type. As with expanders, views must be explicitly imported in order to be considered for such inference. In this way, on the client side views are quite similar to expanders. However, on the implementation side views provide no special support for adaptation. For example, in order to augment a type with new methods, the programmer has to implement a view that explicitly creates a wrapper class for values of that type, along with forwarding methods for all of the original methods of that type. In eJava, these details are taken care of by the compiler. Additionally, Scala does not support any form of overriding for views, so programmers must manually implement run-time type dispatch in order to adapt different subclasses in different ways.

MultiJava's open classes [6] allow new methods to be added to existing classes externally. As in eJava, clients in MultiJava explicitly import any new methods they wish to employ, thereby allowing for modular reasoning. MultiJava does not support the introduction of new superinterfaces to existing classes and hence is not able to express object adapters and related idioms. MultiJava also does not support the introduction of new fields to existing classes. The inclusion of superinterfaces and fields in expanders constitutes a large increase in expressiveness over MultiJava and requires significant new work to support modular typechecking and compilation.

Half \& Half [1] is an extension to Java that supports the ability to add new superinterfaces to existing classes, as well as a form of multiple dispatch. A wrapper strategy similar to the one we describe is used to compile the new language construct. Unlike eJava, Half \& Half does not support the addition of new methods or fields to existing classes. Therefore, a new interface can only be given to an existing class if it already meets all the requirements of that interface.

Several language features, including mixins $[5,13,29]$, traits [28], and parameterized modules [12, 20], support the flexible creation of new classes from existing ones. In contrast, expanders flexibly update existing classes in place. Therefore, expanders can be used to adapt existing objects with new capabilities, while the approaches mentioned above cannot. On the other hand, those language constructs provide fine-grained code reuse across independent classes, while expanders do not. Those languages also support some expressiveness that could be useful to incorporate in expanders. For example, Schärli et al. [28] describe an expressive sublanguage that allows clients to resolve conflicts that may occur when combining multiple traits. As another example, Jiazzi [20] supports a very expressive form of class parameterization, including recursive linking.

There have also been several languages that include a form of virtual types $[32,31]$ and related forms of dependently typed classes [10,11, 21, 23]. These languages allow hierarchies of classes to easily be refined in various ways. Like the languages described above, these approaches allow new hierarchies to be created from existing ones, rather than updating existing hierarchies in place. Therefore, these approaches are not suitable for adapting existing objects to new clients. On the other hand, these approaches allow multiple independent hierarchies to exist in a program, along with a guarantee that instances of the constituent classes will only intermingle with other instances from the same hierarchy.

\section{Conclusions and Future Work}

We have described the expander, a new construct that provides explicit language support for modularly adapting objects in flexible ways to suit the needs of clients. Expanders can be used to implement a variety of recurring idioms in object-oriented software (such as the visitor and adapter design patterns), in a manner that is more declarative, more extensible, and more amenable to static typechecking. We have instantiated our notion of expanders in eJava, an extension to Java. We have formalized a core subset of the eJava language and proven its modular type system sound. We have also implemented eJava via a modular scheme for translating expanders to Java. Lastly, we demonstrated ways in which expanders can provide benefits in practice through two experiments.

The exploratory study in Eclipse pointed out a few limitations of eJava that we plan to address in future work. First, while a visitor can easily inherit code from another visitor, this is not true for expanders. It would be useful to consider a notion of expander inheritance, which would allow a new expander family to be easily derived from an existing expander family. Second, the exploratory study illustrated a potential use for the ability to augment existing classes with new superclasses, in addition to new superinterfaces. We plan to explore this idea to determine if the resulting gain in expressiveness justifies the added complexity. Finally, we plan to extend the eJava language to handle the features in Java 1.5 by porting the eJava compiler to a recently announced extension to Polyglot for Java 1.5 [26].

\section{Acknowledgments}

This material is based upon work supported by the National Science Foundation under Grant Nos. CCF-0427202 and CCF-0545850, as well as by a generous gift from Microsoft Research. Thanks to Craig Chambers, Doug Lea, Stephen Murrell, Yoshiki Ohshima, 
and the anonymous reviewers (of both OOPSLA and ECOOP 2006) for useful feedback on this work.

\section{References}

[1] G. Baumgartner, M. Jansche, and K. Laufer. Half \& Half: Multiple dispatch and retroactive abstraction for Java. Technical Report OSU-CISRC-5/01-TR08, Department of Computer and Information Science, The Ohio State University, revised March 2002.

[2] A. Bergel. Personal communication, Oct. 2005.

[3] A. Bergel, S. Ducasse, and O. Nierstrasz. Classbox/J: Controlling the scope of change in Java. In Proceedings of Object-Oriented Programming, Systems, Languages, and Applications (OOPSLA'05), pages 177-189, New York, NY, USA, 2005. ACM Press.

[4] A. Bergel, S. Ducasse, and R. Wuyts. Classboxes: A minimal module model supporting local rebinding. In Proceedings of JMLC 2003 (Joint Modular Languages Conference), volume 2789 of LNCS, pages 122-131. Springer-Verlag, 2003. Best Award Paper.

[5] G. Bracha and W. Cook. Mixin-based inheritance. In ECOOP/OOPSLA '90, pages 303-311, 1990.

[6] C. Clifton, G. T. Leavens, C. Chambers, and T. Millstein. MultiJava: Modular open classes and symmetric multiple dispatch for Java. In OOPSLA 2000 Conference on Object-Oriented Programming, Systems, Languages, and Applications, Minneapolis, Minnesota, volume 35(10) of ACM SIGPLAN Notices, pages 130-145, Oct. 2000.

[7] C. Clifton, T. Millstein, G. T. Leavens, and C. Chambers. MultiJava: Design rationale, compiler implementation, and applications. ACM Trans. Program. Lang. Syst., 28(3):517-575, 2006.

[8] Eclipse home page. http://www.eclipse.org.

[9] Eclipse Bug System home page. https://bugs.eclipse.org/ bugs.

[10] E. Ernst. Family polymorphism. In Proceedings of the European Conference on Object-Oriented Programming, pages 303-326, 2001.

[11] E. Ernst. Higher-order hierarchies. In Proceedings of the European Conference on Object-Oriented Programming, LNCS, Darmstadt, Germany, July 2003. Springer Verlag.

[12] R. B. Findler and M. Flatt. Modular object-oriented programming with units and mixins. In Proceedings of the ACM SIGPLAN International Conference on Functional Programming (ICFP '98), volume 34(1) of ACM SIGPLAN Notices, pages 94-104. ACM, June 1998.

[13] M. Flatt, S. Krishnamurthi, and M. Felleisen. Classes and mixins. In Conference Record of POPL 98: The 25TH ACM SIGPLAN-SIGACT Symposium on Principles of Programming Languages, San Diego, California, pages 171-183, New York, NY, 1998.

[14] E. Gamma and K. Beck. Contributing to Eclipse: Principles, Patterns, and Plugins. Addison Wesley Longman Publishing Co., Inc., Redwood City, CA, USA, 2003.

[15] E. Gamma, R. Helm, R. E. Johnson, and J. Vlissides. Design Patterns: Elements of Reusable Object-Oriented Software. Addison-Wesley, Massachusetts, 1995.

[16] J. Gosling, B. Joy, G. Steele, and G. Bracha. The Java Language Specification, Third Edition. Addison-Wesley, 2005.

[17] W. Harrison and H. Ossher. Subject-oriented programming (A critique of pure objects). In A. Paepcke, editor, Proceedings ACM Conference on Object-Oriented Programming Systems, Languages, and Applications, pages 411-428. ACM Press, Oct. 1993.
[18] A. Igarashi, B. C. Pierce, and P. Wadler. Featherweight Java: a minimal core calculus for Java and GJ. ACM Transactions on Programming Languages and Systems, 23(3):396-450, May 2001.

[19] G. Kiczales, E. Hilsdale, J. Hugunin, M. Kersten, J. Palm, and W. G. Griswold. An overview of AspectJ. In Proceedings of the 2001 European Conference on Object-Oriented Programming, LNCS 2072, Budapest, Hungary, June 2001. Springer-Verlag.

[20] S. McDirmid, M. Flatt, and W. C. Hsieh. Jiazzi: New-age components for old-fashioned Java. In Proceedings of the OOPSLA '01 conference on Object Oriented Programming Systems Languages and Applications, pages 211-222. ACM Press, 2001.

[21] N. Nystrom, S. Chong, and A. C. Myers. Scalable extensibility via nested inheritance. In OOPSLA '04: Proceedings of the 19th annual ACM SIGPLAN Conference on Object-oriented programming, systems, languages, and applications, pages 99-115. ACM Press, 2004.

[22] N. Nystrom, M. R. Clarkson, and A. C. Myers. Polyglot: An extensible compiler framework for Java. In Proceedings of CC 2003: 12'th International Conference on Compiler Construction. Springer-Verlag, Apr. 2003.

[23] M. Odersky and M. Zenger. Scalable component abstractions. In OOPSLA '05: Proceedings of the 20th annual ACM SIGPLAN conference on Object oriented programming systems languages and applications, pages 41-57, New York, NY, USA, 2005. ACM Press.

[24] H. Ossher, M. Kaplan, A. Katz, W. Harrison, and V. Kruskal. Specifying subject-oriented composition. Theor. Pract. Object Syst., 2(3):179-202, 1996

[25] H. Ossher and P. Tarr. Hyper/J: Multi-dimensional separation of concerns for Java. In Proceedings of the 22nd International Conference on Software Engineering, June 4-11, 2000, Limerick, Ireland, pages 734-737, 2000.

[26] Polyglot for Java 1.5 home page. http://www.sable.mcgill.ca/ jlhotak/polyglot-custom.

[27] The Scala language home page. http://scala.epfl.ch.

[28] N. Schärli, S. Ducasse, O. Nierstrasz, and A. Black. Traits: Composable units of behavior. In Proceedings ECOOP 2003 (European Conference on Object-Oriented Programming), volume 2743 of LNCS, pages 248-274. Springer Verlag, July 2003.

[29] Y. Smaragdakis and D. Batory. Implementing layered designs with mixin layers. In E. Jul, editor, ECOOP '98-Object-Oriented Programming, LNCS 1445, pages 550-570. Springer, 1998.

[30] P. Tarr, H. Ossher, W. Harrison, and J. Stanley M. Sutton. N degrees of separation: multi-dimensional separation of concerns. In ICSE '99: Proceedings of the 21st international conference on Software engineering, pages 107-119, Los Alamitos, CA, USA, 1999. IEEE Computer Society Press.

[31] K. K. Thorup and M. Torgersen. Unifying genericity: Combining the benefits of virtual types and parameterized classes. In R. Guerraoui, editor, Proceedings ECOOP '99, volume 1628 of LNCS, pages 186204, Lisbon, Portugal, June 1999. Springer-Verlag.

[32] M. Torgersen. Virtual types are statically safe. In International Workshop on Foundations of Object-Oriented Languages (FOOL), informal proceedings, Jan. 1998.

[33] A. Warth and T. Millstein. Featherweight eJava. Technical Report CSD-TR-060013, UCLA Computer Science Department, 2006. ftp: //ftp.cs.ucla.edu/tech-report/2006-reports/060013.pdf.

[34] A. K. Wright and M. Felleisen. A syntactic approach to type soundness. Information and Computation, 115(1):38-94, Nov. 1994. 